\title{
QUEEN'S
UNIVERSITY
BELFAST
}

\section{A binary symmetric based hybrid meta-heuristic method for solving mixed integer unit commitment problem integrating with significant plug-in electric vehicles}

Yang, Z., Li, K., Guo, Y., Feng, S., Niu, Q., Xue, Y., \& Foley, A. (2019). A binary symmetric based hybrid metaheuristic method for solving mixed integer unit commitment problem integrating with significant plug-in electric vehicles. Energy, 170, 889-905. https://doi.org/10.1016/j.energy.2018.12.165

Published in:

Energy

Document Version:

Peer reviewed version

Queen's University Belfast - Research Portal:

Link to publication record in Queen's University Belfast Research Portal

\section{Publisher rights}

Copyright 2019 Elsevier Ltd.

This manuscript is distributed under a Creative Commons Attribution-NonCommercial-NoDerivs License

(https://creativecommons.org/licenses/by-nc-nd/4.0/), which permits distribution and reproduction for non-commercial purposes, provided the author and source are cited.

\section{General rights}

Copyright for the publications made accessible via the Queen's University Belfast Research Portal is retained by the author(s) and / or other copyright owners and it is a condition of accessing these publications that users recognise and abide by the legal requirements associated with these rights.

Take down policy

The Research Portal is Queen's institutional repository that provides access to Queen's research output. Every effort has been made to ensure that content in the Research Portal does not infringe any person's rights, or applicable UK laws. If you discover content in the

Research Portal that you believe breaches copyright or violates any law, please contact openaccess@qub.ac.uk. 


\title{
A binary symmetric based hybrid meta-heuristic method for solving mixed integer unit commitment problem integrating with significant plug-in electric vehicles
}

\author{
Zhile Yang ${ }^{\mathrm{a}}$, Kang Li ${ }^{\mathrm{b}}$, Yuanjun Guo ${ }^{\mathrm{a}^{*}}$, Shengzhong Feng ${ }^{\mathrm{a}}$, Qun Niuc, Yusheng Xue ${ }^{\mathrm{d}}$, Aoife Foley \\ a Shenzhen Institute of Advanced Technology, Chinese Academy of Sciences, Shenzhen, Guangdong, \\ 518055, China (Email: zyang07@qub.ac.uk,yi.quo@siat.ac.cn, sz.feng@siat.ac.cn). \\ $b$ School of Electronic and Electrical Engineering, University of Leeds, Leeds, LS2 9JT, UK \\ (Email:k.li01@leeds.ac.uk). \\ 'School of Mechatronic Engineering and Automation, Shanghai Key Laboratory of Power Station \\ Automation Technology, Shanghai University, Shanghai 200072, China (Email: \\ comelycc@hotmail.com). \\ ${ }^{d}$ State Grid Electric Power Research Institute, 210003, Jiangsu, China (Email: xueyusheng@sgepri. \\ sgcc.com.cn). \\ e School of Mechanical and Aerospace Engineering, Queen's University Belfast, Belfast, BT9 5AH, \\ United Kingdom (Email: a.foley@qub.ac.uk).
}

Abstract: Conventional unit commitment is a mixed integer optimisation problem and has long been a key issue for power system operators. The complexity of this problem has increased in recent years given the emergence of new participants such as large penetration of plug-in electric vehicles. In this paper, a new model is established for simultaneously considering the day-ahead hourly based power system scheduling and a significant number of plug-in electric vehicles charging and discharging behaviours. For solving the problem, a novel hybrid mixed coding meta-heuristic algorithm is proposed, where V-shape symmetric transfer functions based binary particle swarm optimization are employed. The impact of transfer functions utilised in binary optimisation on solving unit commitment and plug-in electric vehicle integration are investigated in a 10 unit power system with 50,000 plug-in electric vehicles. In addition, two unidirectional modes including grid to vehicle and vehicle to grid, as well as a bi-directional mode combining plug-in electric vehicle charging and discharging are comparatively examined. The numerical results show that the novel symmetric transfer function based optimization algorithm demonstrates competitive performance in reducing the fossil fuel cost and increasing the scheduling flexibility of plug-in electric vehicles in three intelligent scheduling modes.

Keywords: plug-in electric vehicles, unit commitment, vehicle to grid, symmetric transfer function, binary particle swarm optimisation, meta-heuristic 


\section{Nomenclature}

\begin{tabular}{|c|c|}
\hline$a_{j}, b_{j}$ and $c_{j}$ & Coefficients of fuel cost for unit $j$ \\
\hline$C_{1}$ & Social coefficient in PSO \\
\hline$C_{2}$ & Cognitive coefficient in PSO \\
\hline$C R$ & Crossover rate in SaDE \\
\hline $\mathrm{E}_{E V, \text { total }}$ & Total energy necessity of PEVs \\
\hline$F_{j, t}$ & Fuel cost of unit $j$ at time $t$ \\
\hline$M D T_{j}$ & Minimum down time of unit $j$ \\
\hline$M U T_{j}$ & Minimum up time of unit $j$ \\
\hline$m v_{j, i, G}$ & Mutation vector in SaDE \\
\hline$n$ & Number of units \\
\hline$n s_{1}, n s_{2}$ & Successful times for corresponding variant in SaDE \\
\hline$n f_{1}, n f_{2}$ & Failure times for corresponding variant in SaDE \\
\hline$N p$ & Number of particle \\
\hline$P$ & Probability transfer function in BSPSO \\
\hline$P_{j, \min }$ & Minimum power limits of unit $j$ \\
\hline$P_{j, \max }$ & Maximum power limits of unit $j$ \\
\hline$P_{j, t}$ & Determined power of unit $j$ at time $t$ \\
\hline$P_{D, t}$ & Forecast power demand \\
\hline$P_{P E V, t}$ & Charge or discharging power for PEVs at time $t$ \\
\hline$P_{E V C, t, \max }$ & Maximum charge power for PEVs at time $t$ \\
\hline$P_{E V C, t, \min }$ & Minimum charge power for PEVs at time $t$ \\
\hline$P_{E V D, t, \max }$ & Maximum discharge power from PEVs at time $t$ \\
\hline$P_{E V D, t, \min }$ & Minimum discharge power from PEVs at time $t$ \\
\hline$p_{\text {gbest }}$ & Global best solution in BSPSO \\
\hline$p_{\text {lbest }, i}$ & Local best solution in BSPSO \\
\hline$p_{s}$ & Probability of selection in SaDE \\
\hline $\mathrm{R}_{\text {NThe,t }}$ & Non-thermal power plant reserve amount at time $t$ \\
\hline$R_{\text {The, } t}$ & Thermal plant power plant reserve amount at time $t$ \\
\hline$S U_{C, j}$ & Cold-start cost of unit $j$ at time $t$ \\
\hline$S U_{H, j}$ & Hot-start cost of unit $j$ at time $t$ \\
\hline$S U_{j, t}$ & Start-up cost of unit $j$ at time $t$ \\
\hline$T$ & Total scheduling hours \\
\hline$T_{\text {cold }, j}$ & Cold-start hour of unit $j$ \\
\hline $\operatorname{tr}_{j, i, G}$ & Trail vector in SaDE \\
\hline$T_{O F F_{j, t}}$ & Off-line duration time of unit $j$ \\
\hline $\operatorname{TON}_{j, t}$ & On-line duration time of unit $j$ \\
\hline$u_{j, t}$ & Binary status of unit $j$ at time $t$ \\
\hline$v_{i}$ & Velocity in $i^{\text {th }}$ binary particle at $k^{\text {th }}$ iteration \\
\hline$w$ & Weighting factor \\
\hline$X_{P E V}$ & Charging or discharging power of PEVs \\
\hline$X_{r 1, G}, X_{r 2, G}, X_{r 3, G}$ & Three random particles in SaDE \\
\hline
\end{tabular}

\section{Introduction}

Unit commitment (UC) in power system aims to minimize generation costs by determining the on/off status and power delivered from generation units under various system constraints. It is a large scale complex mixinteger nonlinear problem which has long been a major issue faced by power system operators (Quan et al., 2016). A number of approaches have been proposed including conventional methods, mixed coded meta- 
heuristic methods and hybrid binary meta-heuristic/Lagrangian based methods. Conventional methods see low computational costs for limited size UC problems. Dynamic programming (DP) (Snyder et al., 1987) could quick solve limited dimension UC problem and achieve satisfied results. Lagrangian relaxation (LR) (Jiang et al., 2013) revises the original problem formulation and also obtains the results in a relatively short time period. But they both lack accuracy due to the reformulation aiming for algorithm compatible simplification, as well as endure the 'curse of dimensionality' for large scale scenarios. Mixed coded meta-heuristic algorithms have been proposed and show advantages in the exploitation of high dimensional models. Harmony search (HS) (Kamboj et al., 2015) and particle swarm optimization (PSO) (Shukla and Singh, 2016) hybridized the similar structure of a single heuristic algorithm, whereas the paper (Trivedi et al., 2015) combined the genetic algorithm with differential evolution (hGADE). However, they also endure significant computational costs. Moreover, binary meta-heuristic algorithm such as genetic algorithm (GA) (Kazarlis et al., 1996) and binary particle swarm optimization (BPSO) (Yuan et al., 2009; Zhang et al., 2016) combining the GA and PSO with lambda iteration provide a trade-off between computational cost and accuracy and have been important alternative choices in solving traditional UC problem. However, few publications have utilised V-shape symmetric binary methods which is shown to be promising variants (Mirjalili and Lewis, 2013) for solving the unit commitment problem. In addition, as more participants, such as plug-in electric vehicles, embedded generations and intermittent renewable generations, are emerging in recent years, the UC problem becomes more challenging for optimization algorithms (Quan et al., 2015), which calls for more efficient methods.

A major issue is the scheduling of charging and discharging of plug-in electric vehicles. Generally speaking, electric vehicles (EVs) can be categorized as battery electric vehicles (BEVs), hybrid electric vehicles (HEVs) (non-plug-in), and plug-in hybrid electric vehicles (PHEVs) (Chan, 2007). Considering the battery capacity and common charging necessity, both BEVs and PHEVs are referred as plug-in EVs (PEVs). Due to continual investment in research \& development, the capacity of EV batteries is quickly increasing and has achieved up to $90 \mathrm{kWh}$ for a single vehicle battery pack. On the basis of large battery storage and increasing energy demand of PEVs, some studies have been focused on the utilization of battery capacity of PEVs for system load shifting (Clement-Nyns et al., 2010) (Foley et al., 2013) (Yang et al., 2014), providing vehicle to grid (V2G) service (Kempton and Tomić, 2005), ancillary service (Deilami et al., 2011), power loss minimisation (White and Zhang, 2011), and power reserve (Sanchez-Martin et al., 2015) (Pavić et al., 2015), as well as playing multiple 
roles as energy storages. Interaction between PEVs and Renewable energies have also be considered (Wang et al., 2011) (Dallinger and Wietschel, 2012) to increase both of their penetrations. However, the majority of previous studies schedule PEVs charging/discharging under certain dispatch scenarios or solve the power system scheduling problem using conventional methods or commercial tools (Yang et al., 2015), such as linear programming (Jin et al., 2013; Sundstrom and Binding, 2012), quadratic programming (Jian et al., 2015) and mixed integer linear programing (Liu et al., 2012) (Khodayar et al., 2012) implemented in CPLEX or GAMS. These approaches normally lack flexibility in the problem modelling and require sacrifice the accuracy to satisfy the solvers criteria. The novel optimisation model proposed in the paper is a highly complicated hybrid optimisation model considering the mixed-integer UC system and the flexible scheduling of plug-in electric vehicles along the 24 day-ahead time horizon, which lead to the failures of the classical approaches. This motivates the authors to propose a novel approach to tackle with the intractable task.

In this paper, a novel hybrid meta-heuristic algorithm has been proposed for solving a novel UC problem integrating significant PEVs. The major contributions of the paper are shown below:

1) A novel optimisation model named UCSPEV problem is formulated, flexibly integrating the UC problem with intelligent scheduling of PEV charging/discharging. The significant number of PEVs is modelled as an aggregator and able to provide bidirectional power flows interacting with the power system.

2) A new hybrid meta-heuristic method framework combines binary symmetric PSO (BSPSO) method, a self-adaptive differential evolution ( $\mathrm{SaDE}$ ) algorithm and a lambda iteration method to holistically determine binary status of generators, the commit power of online units as well as the flexible power flow of PEVs for UCSPEV problem.

3) The impact of transfer functions in the binary PSOs on the optimal economic results of both UC problem and UCSPEV has been firstly and comprehensively evaluated.

4) The proposed UCSPEV problem integrated 50,000 PEVs are evaluated in unidirectional power flow scenarios including the G2V scenario and a vehicle to grid (V2G) mode scenario, as well as a bidirectional energy flow scenario combining both G2V/V2G modes. Multiple levels of power reserve are comparatively studied to analyse the system reliability and the economic factor. 
Numerical results confirm that the proposed new hybrid method outperforms existing counterparts in terms of saving fuel and operational cost of UC both with and without PEVs, and the flexible scheduling of PEVs provide potentials to significantly reduce the generation cost. This paper focuses on how a proper optimisation scheduling method could help on the coordinated charging and discharging behaviours of PEVs to reduce the economic cost. The method and idea could easily be transferred into the other grid components including the stochastic RES energy sources.

The rest of the paper is organized as follows: Section 2 formulates the novel UCsPEV problem where the objection function and constraints are provided. The binary symmetric based hybrid meta-heuristic method is then proposed in Section 3, followed by the comprehensive numerical analysis on the evaluation of BSPSO and multiple case studies for the UCSPEV problem addressed in Section 4. Finally, Section 5 concludes the paper.

\section{Problem formulation}

The new model framework integrates thermal UC problem with three scenarios of PEVs as shown in Fig.1. The system operator determines the day-ahead schedule of thermal power plants according to the power demand as well as coordinates the power delivering/receiving to/from the PEV aggregators. Three flexible modes are investigated including a G2V mode, a V2G mode as well as a G2V/V2G bidirectional mode. The G2V mode only considers the PEV aggregator as a dispatchable charging load being determined simultaneously with UC problem. The V2G mode utilizes renewable energy generation to provide the energy necessity of PEVs and takes PEVs aggregator as a virtual power plant which only feeds power back to the grid rather than receives power. In the charging/discharging mode finally, two unidirectional modes are combined. In all three modes, PEV aggregators are designed to possess options for delivering or receiving power to/from the grid. 


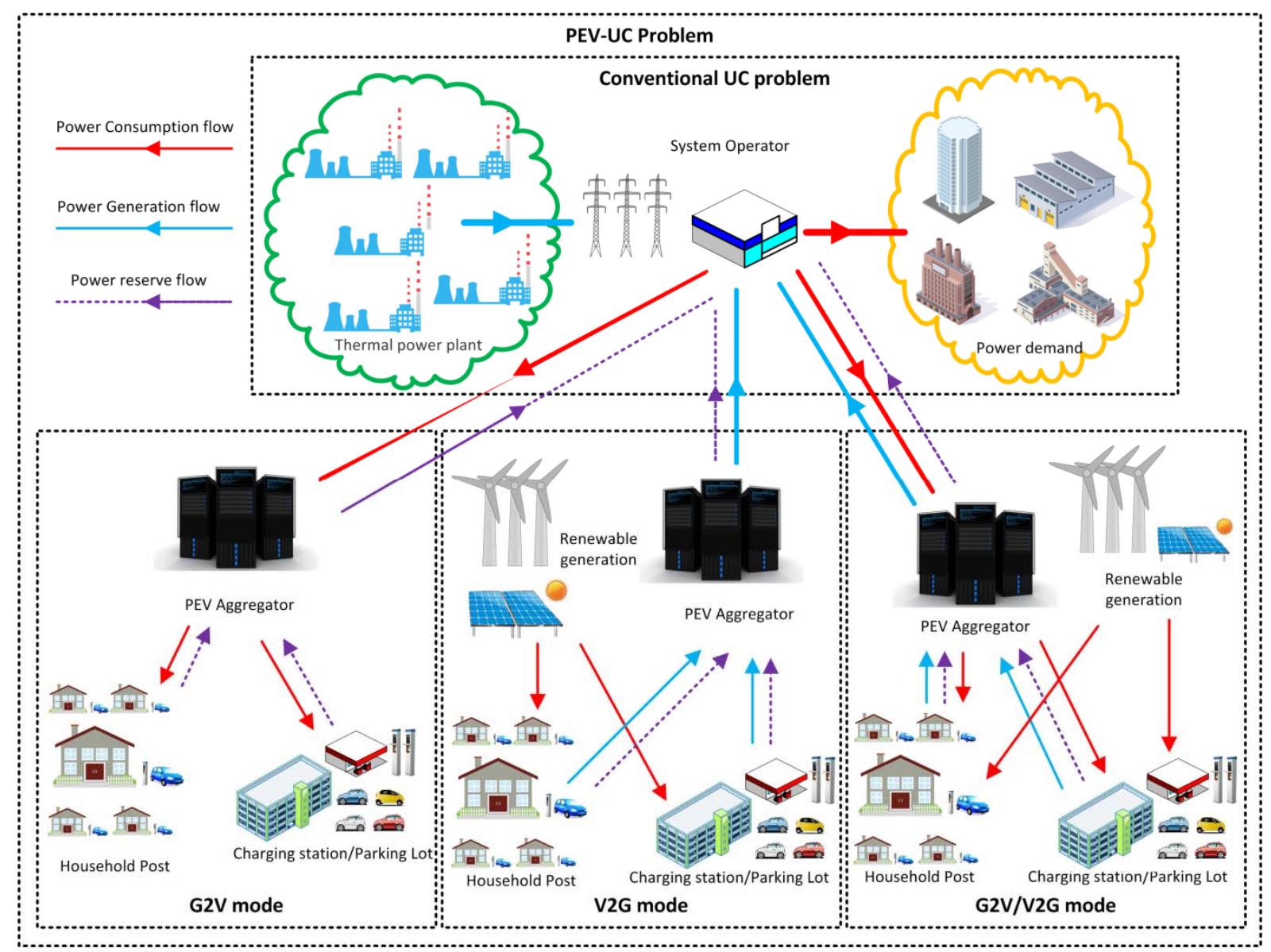

Fig. 1 Framework of UCSPEV problem

The new UCSPEV problem shares the similar mathematical formulation as the traditional UC problem (Kazarlis et al., 1996) in terms of the objective function and system constraints. In addition, several PEV constraints are incorporated into the formulation to model the practical limitations of PEV charging and discharging.

\subsection{Objective function}

The objective function is composed of two parts of economic cost, including fossil fuel and start-up cost.

1) Fuel cost

$$
F_{j, t}\left(P_{j, t}\right)=a_{j}+b_{j} \cdot P_{j, t}+c_{j} \cdot P_{j, t}^{2}
$$

Fuel cost is a quadratic formulation shown in (1) with the $P_{j, t}$ and $F_{j, t}$ denoting the determined power and fuel cost at time $t . a_{j}, b_{j}$ and $c_{j}$ are the fuel cost coefficients.

2) Start-up cost

$$
S U_{j, t}=\left\{\begin{array}{l}
S U_{H, j}, \text { if } M D T_{j} \leq T O F F_{j, t} \leq M D T_{j}+T_{c o l d, j} \\
S U_{C, j}, \text { if } T O F F_{j, t}>M D T_{j}+T_{c o l d, j}
\end{array}\right.
$$


Start-up cost $S U_{j, t}$ is an inevitable cost to 'turn on' an off-line generator. A cold generator is required to be reheated and has a higher cold-start cost $S U_{C, j}$, while hot-start cost is denoted as $S U_{H, j}$. The minimum down time and minimum up time are denoted as $M D T_{j}$ and $M U T_{j}$ for an online unit to be turned off and vice versa. $T_{\text {cold } j \text { j }}$ is the cold-start hour, whereas $T O F F_{j, t}$ is the off-line duration time.

Due to that various types of PEV batteries lead to significant challenges to quantitatively evaluate average battery cost, the battery depletion cost for PEVs are not considered in this paper for simplification. The final objective cost function is modeled as below,

$$
\min \sum_{t=1}^{T} \sum_{j=1}^{n}\left[F_{j}\left(P_{j, t}\right) \cdot x_{j, t}+S U_{j, t} \cdot\left(1-u_{j, t-1}\right) \cdot u_{j, t}\right]
$$

where $u_{j, t}$ denotes the on/off binary status of corresponding generation unit, where the economic cost is determined by $n$ units over $T$ time periods.

\subsection{Constraints}

The proposed UCSPEV problem inherits the system constraints from conventional UC (Ting et al., 2006) such as generation, power demand limit and spinning reserve limits. However, some new items of PEVs are added in the original limits and novel PEVs relevant constraints are modeled. The novel problem formulation, instead of using statistic scenarios based algorithm (Wang et al., 2011), is able to intelligently determine the PEVs power flow along the 24 hour time horizon.

1) Generation limit

Power system Generation limit describe the power capacity of each unit shown as,

$$
u_{j, t} \cdot P_{j, \min } \leq P_{j, t} \leq u_{j, t} \cdot P_{j, \max }
$$

where $P_{j, \min }$ and $P_{j, \max }$ are the minimum and maximum power capacity.

\section{2) Power demand limit}

Power demand limit illustrates the power balance between power generation and user demand. In the UCSPEV problem, the G2V/V2G power are accumulated as parts of power load demand and generation respectively, as shown below,

$$
\sum_{j=1}^{n} P_{j, t} \cdot u_{j, t}=P_{D, t}+P_{P E V, t}
$$


where $P_{D, t}$ is the system load demand, and $P_{P E V, t}$ represents the G2V power delivered to the PEVs from the thermal generation plants or V2G power provided by the PEVs at time $t$ respectively. It should be noted that the PEVs are either serving in G2V mode or V2G mode at one time interval and are not available to be charged and discharged simultaneously. Therefore it is defined that the positive value of $P_{P E V, t}$ is the G2V power and the negative value is $\mathrm{V} 2 \mathrm{G}$ power in this paper.

\section{3) Power reserve limit}

System load prediction may fail to precisely estimate the real system load demand. The power reserve is therefore necessary to provide redundant power to meet the unpredicted demand requirement. Generally speaking, the majority of current power reserve is provided by thermal generation, especially from fast response gas plants during peak load period. Due to the fast response of battery storage, PEVs can potentially provide power reserve and avoid the expensive operational cost caused by frequency switch of thermal plants. The new reserve limit is modelled as follows,

$$
\sum_{j=1}^{n} P_{j, \max } \cdot u_{j, t} \geq P_{D, t}+R_{T h e, t}+R_{N T h e, t}+P_{P E V, t}
$$

where $R_{T h e, t}$ and $R_{N T h e, t}$ are the reserved power provided by thermal plant and non-thermal plant at time $t$. In this paper, the non-thermal plant $R_{N T h e, t}$ is assumed to be from the renewable power generations such as wind and solar. Whereas the intelligent scheduled PEVs, similar to the power demand limit, serves as an extra load (positive value for $P_{P E V, t}$ ) in charging scenarios and an extra generator (negative value for $P_{P E V, t}$ ) in discharging scenarios. Through extra capacity of PEVs may also serve part of the reserve, it is not considered in the intelligent scheduling amount $P_{P E V, t}$ defined in this paper. The system capacity in the specific hour should not be less than the sum of predicted load and power reserve where the capacity is the accumulation of the maximum capacity of online units and the G2V/V2G power.

\section{4) Minimum up/down time limit}

Traditional thermal power generation units especially coal plants endure minimum up and down time as shown,

$$
u_{j, t}=\left\{\begin{array}{l}
1, \text { if } 1 \leq T O N_{j, t-1}<M U T_{j} \\
0, \text { if } 1 \leq T O F F_{j, t-1}<M D T_{j} \\
0 \text { or } 1, \text { otherwise }
\end{array}\right.
$$


where the unit is forced on or off within minimum periods.

5) Charging/Discharging power limit

The PEV charging/discharging power in each hour is limited by the charging/discharging facility and energy necessity. The charging/discharging power limits are denoted as follows,

$$
\sum_{t=1}^{T} P_{P E V, t}=P_{E V, t o t a l}
$$

Charging mode:

$$
P_{E V C, t, \min } \leq P_{P E V, t} \leq P_{E V C, t, \max }
$$

Discharging mode:

$$
P_{E V D, t, \min } \leq P_{P E V, t} \leq P_{E V C, t, \max }
$$

Charging and discharging mode:

$$
P_{E V D, t, \min } \leq P_{P E V, t} \leq P_{E V C, t, \max }
$$

The power necessity $P_{E V, \text { total }}$ is the total power that needs to be charged for the PEVs in a one day time horizon and calculated by the average mileage of commuter vehicles for normal personal use. The charging/discharging facilities constrain the maximum and minimum power of PEV charging/discharging at time $t$. It is assumed in previous study (Saber and Venayagamoorthy, 2011) that PEV charging/discharging is under a fixed rate and the PEV is scheduled by allocation the charging/discharging number of PEVs. Other than this assumption, power from/to PEVs is modelled as real-valued variable in this paper. This is due to that the determined power generated or delivered for a single PEV in an hour horizon is easy to be adjusted through controlling the charging/discharging time period. It is also reported that the majority of vehicles (over $90 \%$ ) among the total numbers are averagely idle or off road along the all day time horizon, and comparatively conservative assumptions have therefore been made that $50 \%$ of the state of charge are available for the V2G service, which is detailed in the Section 4.

\section{Proposed hybrid meta-heuristic approach}


The UCSPEV problem is a complicated mixed-integer NP-hard problem. Comparing with the conventional UC problem, it is further perplexed by the integration of dispatchable PEVs aggregator working in either G2V, V2G or bi-directional modes. For solving the problem, it is necessary to parallel determine the binary on/off status of all units, the real-valued power generation of online units and the real-valued dispatching power of the PEV aggregator. In this section, A V-shape transfer function based hybrid meta-heuristic method, combining a binary symmetric PSO and a SaDE algorithm, associated with lambda iteration method is proposed to solve the UCSPEV problem.

\subsection{Motivation of proposed hybrid methods}

To tackle the UC problem integrated with PEVs, the basic binary PSO and GA has been employed in previous researches in association with (Ahmed Yousuf Saber and Venayagamoorthy, 2010) or without (Talebizadeh et al., 2014) integer PSO, where the charging and discharging numbers of PEVs are scheduled as integer variables. There are several drawbacks for the methods utilised in these studies. Firstly, basic BPSO and GA both endure low convergence speed and are easy to be trapped within local optimum in solving high dimensional problems. Moreover, the distributions of the PEV powers are either randomly or manually allocated into the 24-hour time horizon, which lacks flexibility and efficiency. In addition, the integer PSO proposed in (Ahmed Yousuf Saber and Venayagamoorthy, 2010) does not seem to be effective enough in exploitation ability. To overcome these drawbacks, a hybrid meta-heuristic method has been proposed in this section. A total 5 binary symmetric PSO variants with different transfer functions, motivated by the publication (Mirjalili and Lewis, 2013), are comparatively studied in solving the conventional UC problem to speed up the performance of binary optimisation. Furthermore, one of the best performed real value optimisation algorithms SaDE method is parallel hybridized with BSPSO variants and lambda iteration method to intelligently determine the UCSPEV power distribution, aiming to increase the both exploration and exploitation ability.

\subsection{Binary symmetric particle swarm optimization}

Binary PSO is a popular PSO variant for discrete problems and has been employed for solving the UC problem (Gaing, 2003)(Chen, 2012). The original BPSO maintains a sigmoid probability function to generate new particles from a probability (Gaing, 2003)(Yuan et al., 2011), The probability is determined by the velocities updated as below, 


$$
v_{i}(k+1)=w(k) \cdot v_{i}(k)+C_{1}(k) \cdot \operatorname{rand}_{1} \cdot\left(p_{\text {lbest }, i}-u_{j, t}(k)\right)+C_{2}(k) \cdot \operatorname{rand}_{2} \cdot\left(p_{\text {gbest }}-u_{j, t}(k)\right)
$$

where $v_{i}(\mathrm{k}+1), v_{i}(\mathrm{k})$ and $u_{j, t}(\mathrm{k})$ are the new, original velocities and the binary variables in $i^{\text {th }}$ binary particle at $k^{\text {th }}$ iteration. The $C_{1}(\mathrm{k}), C_{2}(\mathrm{k})$ and $w(\mathrm{k})$ denote the social and cognitive coefficients as well as the weighting factor respectively. $p_{\text {lbest,i }}$ and $p_{g b e s t}$ are the local and global best solutions, both of which are binary ones. In addition, rand $_{1}$ and rand $_{2}$ are random numbers from $(0,1)$ respectively. It is well known that sigmoid probability function is a preliminary non-linear function with low non-linear behaviours. This may lead to slow convergence of the PSO update process. To remedy this drawback, some researchers redesign the transfer functions as the symmetric V-shape.

In this paper, 5 different transfer functions are redesigned as in Table 1, where absolute value operators are utilized to convert the S-shape probability distributions into the symmetric V-shape ones. The value distributions of the probability associated with the corresponding velocity are shown in figure 2 .

Table 1.

5 variants of BSPSO with their different transfer functions

\begin{tabular}{|c|c|}
\hline \multicolumn{2}{|c|}{ Binary symmetric particle swarm optimisation family } \\
\hline Name & Transfer function \\
\hline BSPSO1 & $P(v)=\left|\operatorname{erf}\left(\frac{\sqrt{\pi}}{2} v\right)\right|$ \\
\hline BSPSO2 & $P(v)=|\tanh (v)|$ \\
\hline BSPSO3 & $P(v)=\left|(v) / \sqrt{1+v^{2}}\right|$ \\
\hline BSPSO4 & $P(v)=\left|\frac{2}{\pi} \arctan \left(\frac{\sqrt{\pi}}{2} v\right)\right|$ \\
\hline BSPSO5 & $P(v)=2 \times\left|\frac{1}{1+e^{-v}}-0.5\right|$ \\
\hline
\end{tabular}




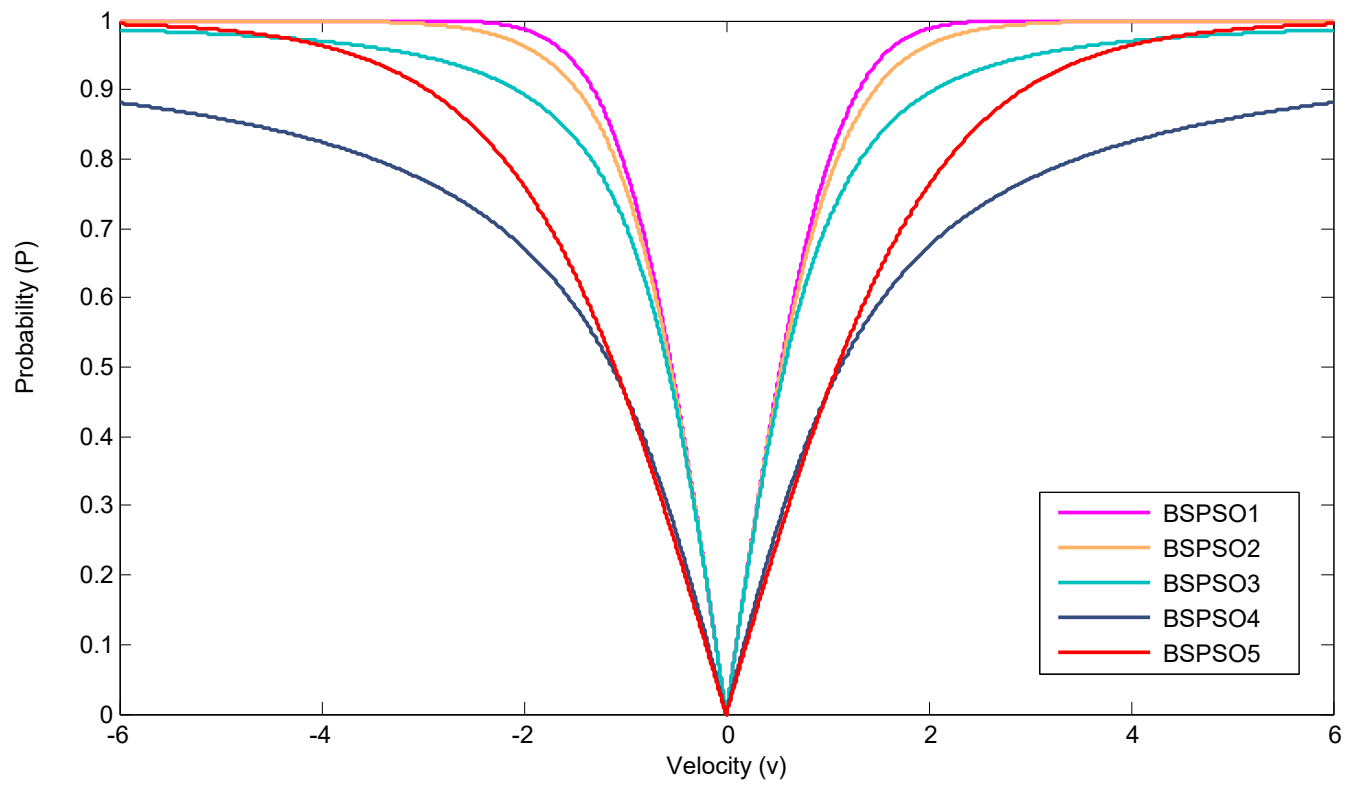

Fig. 2 Symmetric Transfer functions of 5 BSPSO variants

The 5 variants namely BSPSO1 to BSPSO5 are shown in the Table 1 . The given 5 probability transfer functions for BSPSO variants show different distributions along the velocity change. The variant BSPSO1 starts to quickly descend within the velocity range of $[-2,2]$, while the BSPSO4 sees low dropping speed and remains less than 0.9 near the boundary $[-6,6]$. The new population of binary variable $u_{j, t}$ is generated as:

$$
u_{j, t}(k+1)= \begin{cases}1, & \text { if } \text { rand }_{3}<P\left(v_{i}(k+1)\right) \\ 0 & \text { otherwise }\end{cases}
$$

This algorithm variant is named as binary symmetric PSO (BSPSO), and rand $_{3}$ denotes another random number between $(0,1)$. Note that the state-of-the-art binary optimisation based algorithms such as BPSO (Yuan et al., 2011), BGSA (Yuan et al., 2014) and BTLBO (Akhlaghi et al., 2014) only utilised one transfer function for solving UC and other problems. It is therefore worth to comparatively study the impact of the transfer functions in solving the UC problems, and the best performed one will be further utilised to hybridize with other methods and solve the more complicated UCSPEV problem.

\subsection{Self-adaptive differential evolution}

Differential evolution (DE) is another popular heuristic optimization method and has the advantage in various variants candidates for different optimization tasks (Das and Suganthan, 2011). The self-adaptive differential evolution (Qin and Suganthan, 2005) is one of a well-balanced structure which could combine several DE 
variants. In this paper, two DE variants rand/1/bin and current to best/2/bin are embedded in the SaDE structure and adopted to optimize the PEV charging/discharging power. The selection of the variants is determined by the probability $p_{s}$. The process of the SaDE algorithm is denoted as below,

$$
\begin{gathered}
M V_{i, G}= \begin{cases}X_{r 1, G}+F \cdot\left(X_{r 2, G}-X_{r 3, G}\right), & \text { if } p_{s}<p_{1} \\
X_{i, G}+F \cdot\left(X_{\text {best }, G}-X_{i, G}\right)+F \cdot\left(X_{r 1, G}-X_{r 2, G}\right), & \text { otherwise }\end{cases} \\
p_{1}=\frac{n s_{1} \cdot\left(n s_{2}+n f_{2}\right)}{n s_{2} \cdot\left(n s_{1}+n f_{1}\right)+n s_{1} \cdot\left(n s_{2}+n f_{2}\right)} \\
\operatorname{tr}_{l, i, G}=\left\{\begin{array}{c}
m v_{l, i, G}, \quad \text { if } \operatorname{rand}_{4}<C R \text { or } l=l_{\text {rand }} \\
x_{l, i, G}, \quad l=1,2, \ldots, n
\end{array}, \quad\right. \text { otherwise } \\
X_{i, G+1}=\left\{\begin{array}{cc}
T R_{i, G}, & \text { if } f\left(U_{i, G}\right)<f\left(X_{i, G}\right) \\
X_{i, G} & \text { otherwise }
\end{array}\right.
\end{gathered}
$$

where $M V_{i, G}$ and $F$ are the mutation vector and factor. $X_{r 1, G}, X_{r 2, G}, X_{r 3, G}, X_{b e s t, G}$ and $X_{i, G}$ are three random particles, the best and updated candidates. The determined probability $p_{s}$ is a constant and when it is less than $p_{1}$, the exploitation variant rand/1/bin is selected, and vice versa. The self-adaptive behaviours are adjusted by the $p_{1}$. In (15) $n s_{1}, n s_{2}$ and $n f_{1}, n f_{2}$ represent the successful and failure times for corresponding variant. Moreover, $\operatorname{tr}_{j, i, G}, m v_{j, i, G}$ and $x_{j, i, G}$ representing the trail vector, mutation vector and original vector and the crossover rate is denoted as $C R$. The updated strategy is eventually determined by the equation (17).

\subsection{Proposed hybrid algorithm for the new problem}

In this paper, the binary algorithm BSPSO and the real-valued algorithm SaDE are combined to solve the UCSPEV problem. The whole optimization process of the proposed algorithm is illustrated in the figure 3 . There are five steps in the proposed algorithm for solving the UCSPEV problem. 


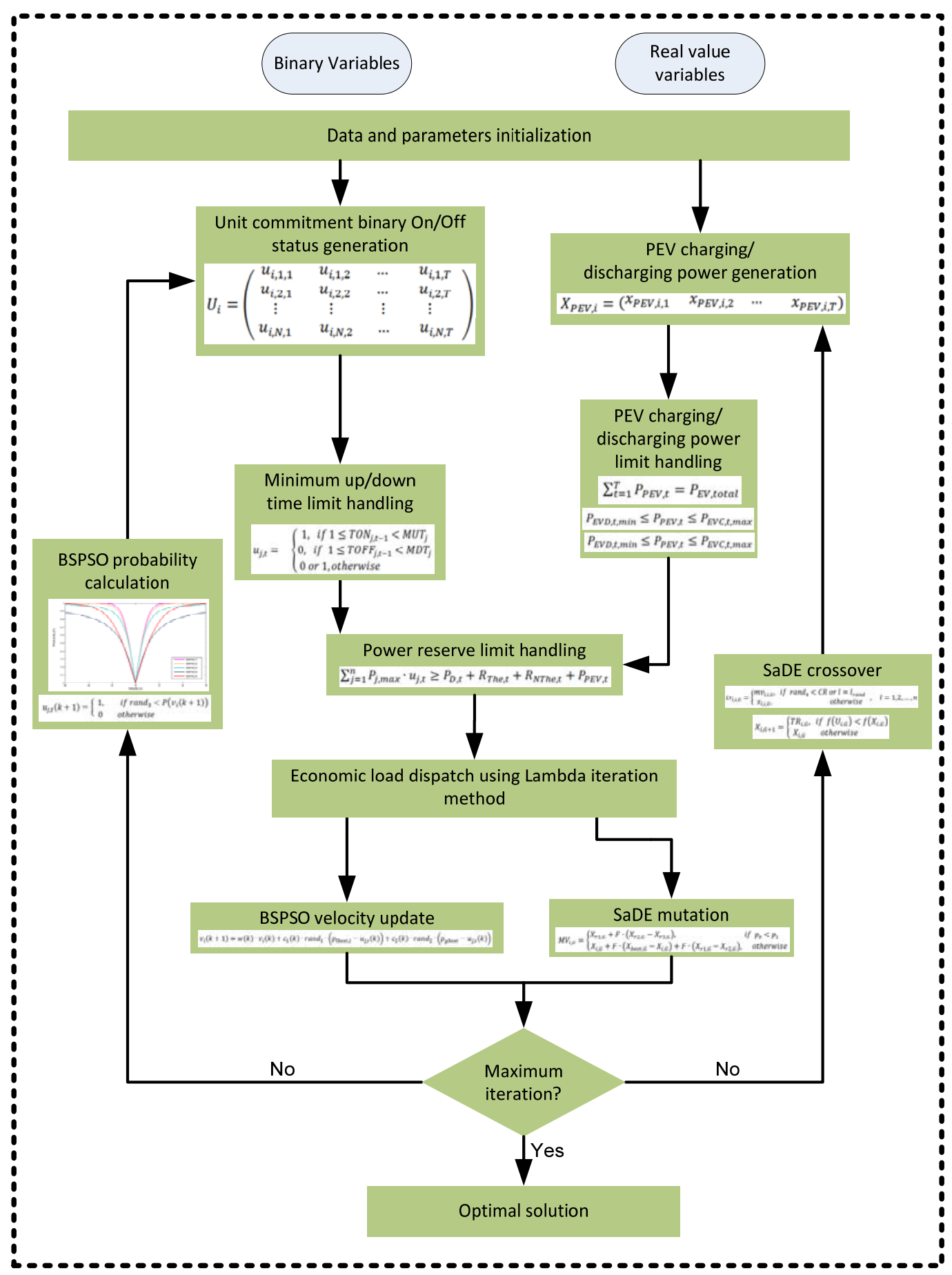

Fig. 3 Optimization process of the proposed hybrid meta-heuristic method for the UCSPEV problem

Step 1: Initialization. In this step, the adopted power system data as well as PEV data including maximum charging power and total power necessity are accessed in the optimization procedure. Algorithm related parameters are initially configured and a hybrid coding particle $U_{i}$ is shown as (18). The $u_{i, 1,1}$ to $u_{i, N, T}$ are binary variables illustrating the online/offline status of all the $N$ units along $T$ time periods. $X_{P E V, 1}$ to $X_{P E V, T}$ are real value 
charging or discharging power of PEVs in each time slots. $N p$ is the number of particle in an optimising population.

$$
\begin{gathered}
U_{i}=\left(\begin{array}{cccc}
u_{i, 1,1} & u_{i, 1,2} & \ldots & u_{i, 1, T} \\
u_{i, 2,1} & u_{i, 2,2} & \ldots & u_{i, 2, T} \\
\vdots & \vdots & \vdots & \vdots \\
u_{i, N, 1} & u_{i, N, 2} & \ldots & u_{i, N, T}
\end{array}\right) \quad i=1,2, \ldots, N p \\
X_{P E V, i}=\left(\begin{array}{lllll}
x_{P E V, i, 1} & x_{P E V, i, 2} & \ldots & \left.x_{P E V, i, T}\right) & i=1,2, \ldots, N p
\end{array}\right.
\end{gathered}
$$

Step 2: Solutions Generation. This step will generate a new iteration of solutions including both binary and real value variables. The binary variables are generated from the equation (14)-(15) by probability functions and the PEV variables are updated according to (16)-(17) where the new trial vector will be tested and adopted.

Step 3: Constraints handling. A number of constraints remain to be handled in the whole procedure. Power system constraints and PEV constraints are handled affiliated to step 2 where the solutions are generated. In addition, the minimum up/down time limit (7) is first handled by the method proposed in (Jeong et al., 2010) through an efficient heuristic based check. Then, PEV limits (8)-(11) are tackled where a special priority list is established for the instruction of distributing PEV power illustrated in next section. Finally, the power reserve limit (6) is handled by the iterations based test, where both binary and real valued based variables are systematically considered.

Step 4: Economic load dispatch (ELD). In this step, a lambda iteration method is applied to solve the ELD subproblem (Yuan et al., 2014), where the limits (4) and (5) are relaxed and the proper solution are obtained in the iteration based update of a lambda value.

Step 5: Variable updates. In this parallel based algorithm procedure, the binary variables are updated in BSPSO by the equation (12) - (13) and real valued solutions are regenerated by SaDE based on (14)-(17). Such process will continue and go back to Step 2 unless achieving the maximum iteration number.

It could be concluded from figure 3 that the hybrid method combines the binary and continuous metaheuristic method and merges the constraints by heuristic handling and lambda iteration approaches. Under such algorithm framework, the evolutionary coordination of the both components is crucial in obtaining high performance results. Given that UC problem is still the backbone of the novel formulated UCsPEV problem, 
binary algorithm BSPSO variants are worth to be evaluated first and a proper variant should be well addressed, then the continuous optimisation SaDE could be complemented in the full problem analysis. The SaDE method has the self-adaptive ability and is able reasonably following up the evolutionary process of BSPSO based binary method. All the numerical evaluation is conducted in the following Section 4.

\section{Numerical results and scenarios analysis}

In this section, the proposed BSPSO algorithm is tested in Case 1 first. It is implemented on a standard 10-unit UC problem which is shown in (Ting et al., 2006) without PEV integration. Then, three different PEVs integration scenarios including charging-only mode, discharging-only mode and charging/discharging intelligent switching mode are studied respectively in Case 2 to Case 4 to comprehensively evaluate the economic impact of PEVs on the power system. To compare the performance of proposed algorithm, 50,000 PEVs are assumed to be integrated in the system as related in (A.Y. Saber and Venayagamoorthy, 2010) (Saber and Venayagamoorthy, 2012) (Saber and Venayagamoorthy, 2011) where the battery average capacity is presumed to be $15 \mathrm{kWh}$. In each mode, different rates of the power reserves are assumed which are provided by thermal plants, with zero, $5 \%$ and $10 \%$ respectively. The unsatisfied reserves are supposed to be supported by renewable energies. All the cases are simulated in an Intel(R) Core(TM) i7-3537U CPU @ 2.50GHz PC and the Matlab (R) 2017a software platform.

\subsection{Case 1: 10-unit only}

In Case 1 (C1), the BSPSO variants associated with the lambda iteration method is used to solve this benchmark case. In terms of the parameter settings for the algorithm, the number of particles in a population is 20 and the maximum iteration is 1000, which aligns other publications (Ji et al., 2014) (Talebizadeh et al., 2014), (Ting et al., 2006). The maximum and minimum of velocity is $[-6,6]$, and the weighting factor $w$ initially decreases from 0.9 to 0.4 . The learning factors $c_{1}$ and $c_{2}$ are chosen 2 and 2 respectively. The spinning reserve is $10 \%$ of power demand. All methods compared in this study are tested in 30 independent runs. The GA (Kazarlis et al., 1996), original binary PSO (BPSO) (Gaing, 2003), a hybrid PSO (HPSO) (Ting et al., 2006), a QPSO (Jeong et al., 2010), a BGSA (Yuan et al., 2014), a GA-LR method (Talebizadeh et al., 2014) and a binary-realcoded GA (brGA) (Datta, 2013), a fuzzy quantum computation-based thermal unit commitment (FQEA) (Chakraborty et al., 2011), a harmony search algorithm (HAS) (Afkousi-Paqaleh et al., 2010), an advanced fuzzy 
controlled binary PSO (AFCBPSO) (Chakraborty et al., 2012), a particle swarm-based simulated annealing algorithm (PSO-B-SA) (Sadati et al., 2007), a quantum inspired evolutionary algorithm (QEA-UC) (Chung et al., 2011), a gravitational search algorithm (GSA) (Roy, 2013), an A. SMP (Khanmohammadi et al., 2010) and a hybrid harmony search method HHS (Kamboj et al., 2015) are selected to comparatively study the performance of the proposed method all under the same test parameters configurations, with the best value, worst value, mean value and standard deviations for each method being listed in Table 2. Note that some original publications do not provide all the results value and the missing parts are denoted as the marker ' - '. To evaluate the performance of the proposed method, the student T-Test (Chen et al., 2015), which is an important statistical methodology, has been utilized and the corresponding scores are also illustrated in the Table 2.

It could be observed from Table 2 that the proposed BSPSO acquires the same or similar best result with BGSA, HPSO, brGA and many other methods at a cost of $563,937 \$ /$ day (some are illustrated as $563,938 \$ /$ day in ceil scheme), which is also the best result among the state-of-the-art UC results. The worst result of BSPSO variants is $563,977 \$ /$ day achieved by BSPSO2-5, being the same as QPSO and the lowest compared with other algorithms. In terms of the mean cost, the variants BSPSO4 and 5 show the advantage of stable searching performance and outperforms all the other counterparts with the mean value of $563,964 \$ /$ day and 563,960 $\$ /$ day respectively. The standard deviations of the 5 BSPSO variants are much smaller than all the other methods with the values of $0.001 \%$ to $0.003 \%$, showing high stability in seeking the optimum. In terms of the T-test comparison, once the T score is over 2 (e.g. P score is less than 0.05), statistical significance between the two competitors could be recognised. The best performed BSPSO5 variant is taken as the benchmark and compare it with all the other counterparts. It could be observed from the Table 2 that the BSPSO5 candidate significantly outperforms 4 out of all the 6 competitors. The advantage of the proposed method is due to the adoption of the V-shape symmetric transfer function. The original unsymmetrical sigmoid transfer function of BPSO has difficulties in determining the binary status in the near zero position because of the half-half possibility, whereas symmetric transfer functions exert higher pressures for the probability values in determining the binary decision variables and therefore speeding up the evolutionary convergence. The transfer function of BSPSO 5 has a reasonable decreasing curve, which match the problem most and produce the best results. 
Table 2.

Simulation results in $\mathrm{C} 1$

\begin{tabular}{|c|c|c|c|c|c|c|}
\hline \multirow{2}{*}{ Method } & \multicolumn{3}{|c|}{ Cost (\$/day) } & \multirow{2}{*}{$\begin{array}{l}\text { Std } \\
(\%)\end{array}$} & \multicolumn{2}{|c|}{ T-Test } \\
\hline & Best & Worst & Mean & & T Score & P Score \\
\hline GA (Kazarlis et al., 1996) & 565,852 & 570,032 & - & - & - & - \\
\hline BPSO (Gaing, 2003) & 565,804 & 567,251 & 566,992 & 0.27 & 10.8465 & 0.0000 \\
\hline HPSO (Ting et al., 2006) & 563,942 & 565,785 & 564,772 & - & - & - \\
\hline QPSO (Jeong et al., 2010) & 563,977 & 563,977 & 563,977 & - & - & - \\
\hline BGSA (Yuan et al., 2014) & 563,937 & 564,241 & 564,031 & 0.05 & 1.3765 & 0.1740 \\
\hline GA-LR (Talebizadeh et al., 2014) & - & - & 564,703 & - & - & - \\
\hline brGA (Datta, 2013) & 563,938 & 564,253 & 564,088 & - & - & - \\
\hline FQEA (Chakraborty et al., 2011) & 563,942 & - & - & - & - & - \\
\hline $\begin{array}{l}\text { HAS (Afkousi-Paqaleh et al., } \\
\text { 2010) }\end{array}$ & 563,977 & - & 564,168 & - & - & - \\
\hline $\begin{array}{l}\text { AFCBPSO (Chakraborty et al., } \\
\text { 2012) }\end{array}$ & 563,947 & 565,002 & 564,285 & - & - & - \\
\hline PSO-B-SA (Sadati et al., 2007) & 563,938 & 564,985 & 564,115 & - & - & - \\
\hline QEA-UC (Chung et al., 2011) & 563,938 & 564,711 & 564,012 & - & - & - \\
\hline GSA (Roy, 2013) & 563,938 & 564,241 & 564,008 & - & - & - \\
\hline $\begin{array}{l}\text { A. SMP (Khanmohammadi et al., } \\
\text { 2010) }\end{array}$ & 563,937 & 564,320 & 564,040 & - & - & - \\
\hline HHS (Kamboj et al., 2015) & 563,937 & 563,995 & 563,965 & - & - & - \\
\hline BSPSO1 & 563,977 & 564,018 & 563,980 & 0.002 & 5.4100 & 0.0000 \\
\hline BSPSO2 & 563,937 & 563,977 & 563,976 & 0.001 & 4.8962 & 0.0000 \\
\hline BSPSO3 & 563,937 & 563,977 & 563,973 & 0.002 & 3.4900 & 0.0009 \\
\hline BSPSO4 & 563,937 & 563,977 & 563,964 & 0.003 & 0.9113 & 0.3659 \\
\hline BSPSO5 & 563,937 & 563,977 & 563,960 & 0.003 & - & - \\
\hline
\end{tabular}

The figure 4 shows the average convergence process of 30 different runs for the five BSPSO variants. It depicts that the five algorithms converge within around 200 iterations, and the BSPSO5 achieves the optimum faster than other counterparts, within only around 120 iterations. Furthermore, the figure 5 illustrates the optimum distributions of the 5 BSPSO variants of 30 different runs. 


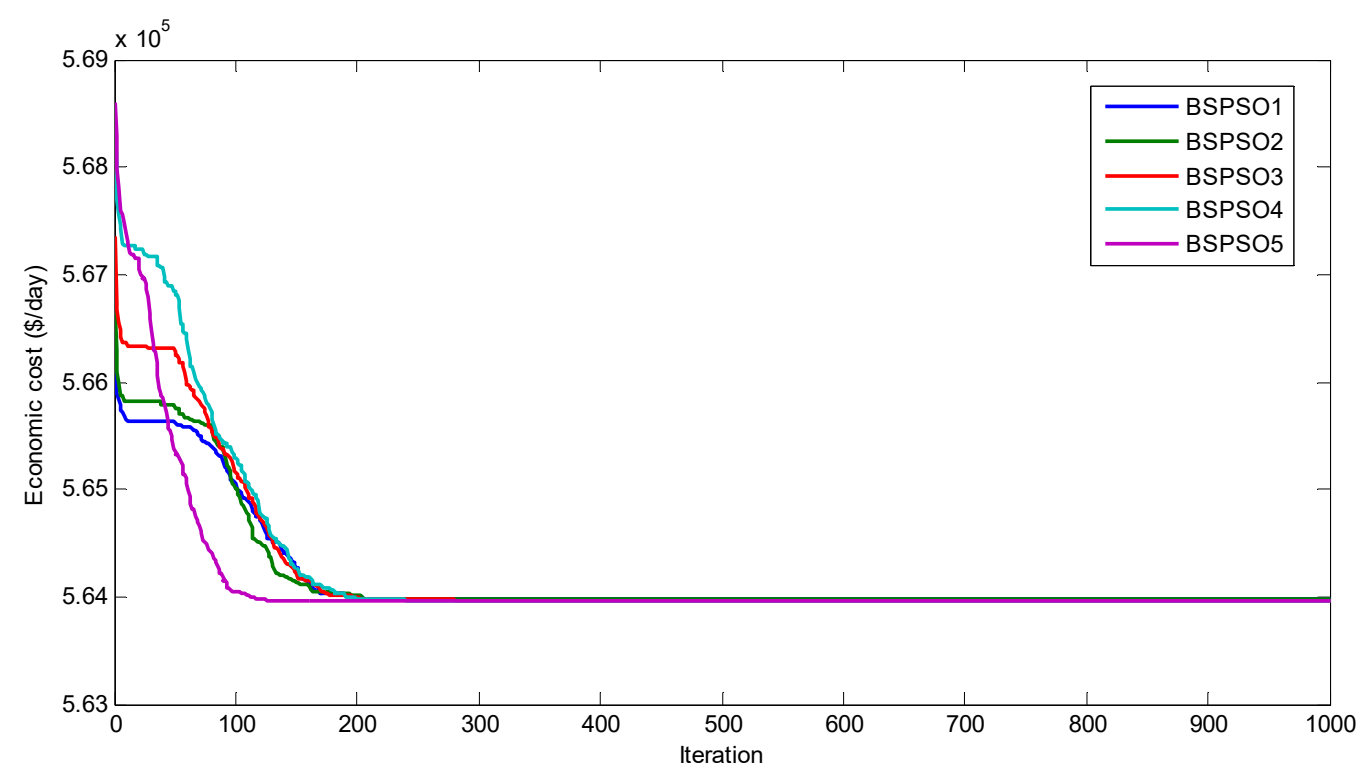

Fig. 4 Convergence process of 5 BSPSO variants on C1

All the results achieved by the 5 BSPSO variants are three values: $563,937 \$ /$ day, $563,977 \$ /$ day and $564,018 \$$ day. It could be observed from the figure 5 that BSPSO5 performs the best in the results distributions, achieving 13 times of the best results 563,937\$/day among the 30 runs, followed by BSPSO 4 which got 10 times best results. Over 25 runs of BSPSO1-BSPSO3 algorithms achieved the results 563,977\$/day, and only BSPSO1 achieved the $564,018 \$$ /day twice.

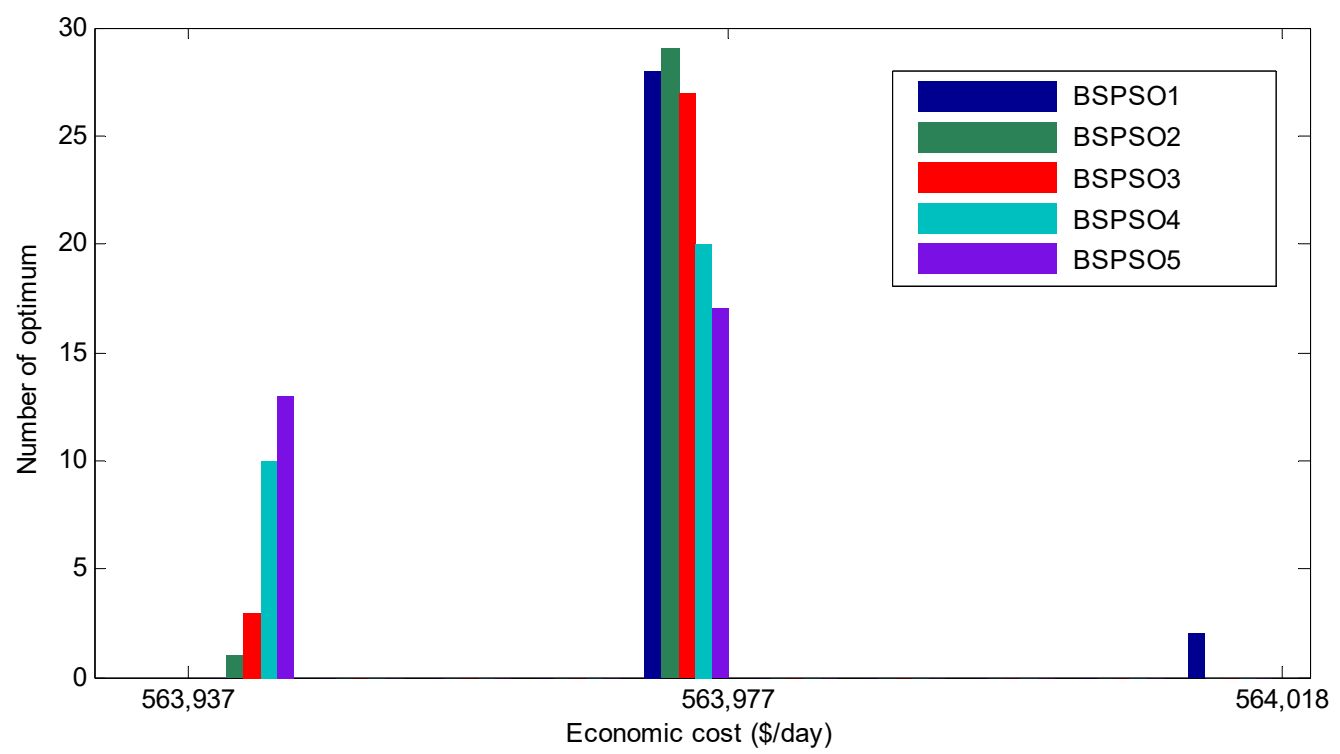

Fig. 5 Result distributions for the BSPSO variants

It could be observed from the results achieved form C1 that all the five BSPSO variants show competitive performance in solving conventional UC problem and the BSPSO5 performs the best. It is therefore utilized to 
work together with real value optimization methods to solve the UCSPEV problem, considering multiple scenarios of PEVs integration.

\subsection{Case 2: 10 unit with plug-electric vehicles charging-only}

There are inevitable interactions between PEV charging and the power system. Intelligent charging helps to relieve the peak load for reducing the expensive fuel cost and fills the valley load for avoiding frequent start-up or shut-down of the thermal power plants. A charging-only scenario is studied in this case to analyse the economic impact of intelligent charging on the power system with 50,000 PEVs integrated in the 10 unit system benchmark. It is assumed according to (A.Y. Saber and Venayagamoorthy, 2010), an average distance of 32.88 miles/day for each PEV with corresponding $8.22 \mathrm{kWh} /$ day energy consumed. Therefore $411 \mathrm{MWh} / \mathrm{day}$ PEV daily charging load needs to be intelligently distributed within a 24-hour time horizon. A maximum charging power is assumed to be $34.25 \mathrm{MW}$ each hour. The proposed meta-heuristic hybrid method combining BSPSO variants and SaDE algorithms is applied to solve this problem.

A priority list shown as Table 3 is applied to provide reference for power distribution of PEVs to handle the PEVs energy limit (8)-(11). The priority list is in ascending orders of predicted load, through which the extra PEV load will be accumulated first for the time period of high priority. To be specific, in the PEVs charging load allocation, the meta-heuristic optimisation method will generate a population of random charging solutions and handle the total charging amount according to the priority charging list, e.g. the lack of total power amount will be preferentially allocated to the valley hours to reduce the potential cost.

Table 3.

The priority list of PEV charging dispatch

\begin{tabular}{|c|c|c|c|c|c|c|c|c|c|c|c|c|}
\hline Priority & $\mathbf{1}$ & $\mathbf{2}$ & $\mathbf{3}$ & $\mathbf{4}$ & $\mathbf{5}$ & $\mathbf{6}$ & $\mathbf{7}$ & $\mathbf{8}$ & $\mathbf{9}$ & $\mathbf{1 0}$ & $\mathbf{1 1}$ & $\mathbf{1 2}$ \\
\hline Hour & $1: 00$ & $2: 00$ & $24: 00$ & $3: 00$ & $23: 00$ & $4: 00$ & $5: 00$ & $17: 00$ & $16: 00$ & $6: 00$ & $18: 00$ & $22: 00$ \\
\hline $\begin{array}{c}\text { Load } \\
\text { (MW) }\end{array}$ & 700 & 750 & 800 & 850 & 900 & 950 & 1000 & 1000 & 1050 & 1100 & 1100 & 1100 \\
\hline Priority & $\mathbf{1 3}$ & $\mathbf{1 4}$ & $\mathbf{1 5}$ & $\mathbf{1 6}$ & $\mathbf{1 7}$ & $\mathbf{1 8}$ & $\mathbf{1 9}$ & $\mathbf{2 0}$ & $\mathbf{2 1}$ & $\mathbf{2 2}$ & $\mathbf{2 3}$ & $\mathbf{2 4}$ \\
\hline Hour & $\mathbf{7 : 0 0}$ & $\mathbf{8}: 00$ & $15: 00$ & $19: 00$ & $9: 00$ & $14: 00$ & $21: 00$ & $10: 00$ & $13: 00$ & $20: 00$ & $11: 00$ & $12: 00$ \\
\hline $\begin{array}{c}\text { Load } \\
\text { (MW) }\end{array}$ & 1150 & 1200 & 1200 & 1200 & 1300 & 1300 & 1300 & 1400 & 1400 & 1400 & 1450 & 1500 \\
\hline
\end{tabular}

Algorithm parameters tuning are important to the performance and required to be done before the application. The benchmark test C1 has shown the reasonably good performance for the BSPSO parameter settings. In order to seek for the proper configurations of SaDE algorithm parameters, 100 parameter 
combinations tests of UCSPEV problem case $210 \%$ thermal power reserve scenario, with mutation factor $F$ and crossover rate $C R$ both ranging from 0.1 to 0.9 , are implemented respectively. In each combination test, the maximum iteration of the algorithm is set as 200 and 10 different runs are implemented to eliminate the randomness. The best performed variant BSPSO5 on the benchmark test $\mathrm{C} 1$ is adopted in these tests to aid the parameter section for the SaDE method. The mean and best results of each parameter combinations tests are shown in Table 4.

Table 4.

SaDE parameter tuning $(\$ /$ day $)$

\begin{tabular}{|c|c|c|c|c|c|c|c|c|c|c|}
\hline \multicolumn{2}{|c|}{ Parameter settings } & $C R=0.1$ & $\mathrm{CR}=0.2$ & $C R=0.3$ & $C R=0.4$ & $C R=0.5$ & $\mathrm{CR}=0.6$ & $C R=0.7$ & $\mathrm{CR}=0.8$ & $C R=0.9$ \\
\hline \multirow{2}{*}{$F=0.1$} & Best & 571,796 & 571,782 & 571,504 & 571,792 & 571,651 & 571,697 & 571,625 & 571,618 & 572,148 \\
\hline & Mean & 572,301 & 572,028 & 572,171 & 572,265 & 572,415 & 572,429 & 572,550 & 572,640 & 572,991 \\
\hline \multirow{2}{*}{$F=0.2$} & Best & 571,680 & 571,449 & 571,474 & 571,484 & 571,491 & 571,578 & 571,557 & 571,573 & 571,782 \\
\hline & Mean & 571,810 & 571,669 & 571,804 & 571,858 & 572,091 & 572,105 & 572,428 & 572,495 & 573,068 \\
\hline \multirow{2}{*}{$F=0.3$} & Best & 571,430 & 571,438 & 571,436 & 571,370 & 571,505 & 571,482 & 571,709 & 571,490 & 571,893 \\
\hline & Mean & 571,616 & 571,688 & 571,666 & 571,732 & 571,829 & 571,950 & 572,414 & 572,121 & 572,724 \\
\hline \multirow{2}{*}{$F=0.4$} & Best & 571,389 & 571,339 & 571,445 & 571,347 & 571,429 & 571,707 & 571,521 & 571,432 & 571,433 \\
\hline & Mean & 571,517 & 571,474 & 571,767 & 571,522 & 571,830 & 572,060 & 572,011 & 571,786 & 572,142 \\
\hline \multirow{2}{*}{$F=0.5$} & Best & 571,347 & 571,329 & 571,330 & 571,310 & 571,326 & 571,353 & 571,345 & 571,378 & 571,457 \\
\hline & Mean & 571,442 & 571,358 & 571,465 & 571,543 & 571,476 & 571,722 & 571,773 & 571,768 & 571,800 \\
\hline \multirow{2}{*}{$F=0.6$} & Best & 571,325 & 571,320 & 571,328 & 571,309 & 571,323 & 571,316 & 571,329 & 571,356 & 571,424 \\
\hline & Mean & 571,339 & 571,340 & 571,344 & 571,385 & 571,372 & 571,434 & 571,467 & 571,619 & 571,818 \\
\hline \multirow{2}{*}{$F=0.7$} & Best & 571,329 & 571,306 & 571,311 & 571,331 & 571,308 & 571,321 & 571,313 & 571,312 & 571,304 \\
\hline & Mean & 571,352 & 571,333 & 571,336 & 571,341 & 571,401 & 571,372 & 571,380 & 571,383 & 571,394 \\
\hline \multirow{2}{*}{$F=0.8$} & Best & 571,341 & 571,327 & $\underline{571,296}$ & 571,305 & 571,341 & 571,346 & 571,322 & 571,354 & 571,337 \\
\hline & Mean & 571,358 & 571,341 & 571,337 & 571,350 & 571,368 & 571,373 & 571,360 & 571,382 & 571,400 \\
\hline \multirow{2}{*}{$F=0.9$} & Best & 571,325 & 571,320 & 571,328 & 571,337 & 571,334 & 571,340 & 571,363 & 571,355 & 571,361 \\
\hline & Mean & 571,339 & 571,340 & 571,344 & 571,354 & 571,357 & 571,376 & 571,387 & 571,408 & 571,398 \\
\hline
\end{tabular}

It could be observed from the Table 4 that the best result for the case $210 \%$ thermal power reserve is $571,296 \$$ day achieved by the parameter combination $C R=0.3 \quad F=0.8$. Though the mean value of this combination is outperformed by the settings $C R=0.2 F=0.7$, it is selected as the best parameter settings and used as the standard parameter configuration of all SaDE relevant methods for the rest of this paper. Table 6 
compares the original BPSO and all BSPSO variants hybrid with SaDE. The tests are employed again on the C2 $10 \%$ thermal power reserve scenario with 10 independent runs for each algorithm and 200 maximum iteration number.

Table 5.

Results comparison of different BPSO variants (\$/day)

\begin{tabular}{|c|c|c|c|c|c|}
\hline \multirow{2}{*}{ Scenarios of PSO algorithms } & \multicolumn{4}{|c|}{ Cost (\$/day) } & \multirow{2}{*}{ Time (s) } \\
\hline & Best & Worst & Mean & Std(\%) & \\
\hline BPSO+SaDE & 572,539 & 573,868 & 573,286 & 0.080 & 57.1 \\
\hline BSPSO1+SaDE & 571,857 & 573,171 & 572,421 & 0.079 & 68.6 \\
\hline BSPSO2+SaDE & 571,820 & 573,100 & 572,721 & 0.070 & 61.5 \\
\hline BSPSO3+SaDE & 571,502 & 572,578 & 572,077 & 0.071 & 66.0 \\
\hline BSPSO4+SaDE & 571,533 & 573,867 & 572,279 & 0.127 & 62.2 \\
\hline BSPSO5+SaDE & 571,296 & 571,383 & 571,337 & 0.005 & 60.3 \\
\hline
\end{tabular}

The best, worst and mean values associated with standard deviations and average computational times of different BPSO based algorithms performance tests are illustrated in Table 5 . It could be observed that the original BPSO see shortest computational time 57.1 seconds but outperformed by all the BSPSO based algorithms in the result comparisons. Among the BSPSO variants, the BSPSO5 achieved the best results in best, worst and mean values as well as standard deviation, using the comparatively low time cost 60.3 seconds. BSPSO3 ranks the second on all factors and BSPSO4 ranks the third among the best values comparison but sees high unstable solution quality in comparing the worst values and standard deviations.

Table 6.

Results comparison of different DE variants (\$/day)

\begin{tabular}{|c|c|c|c|c|c|}
\hline \multirow{2}{*}{ Scenarios of DE algorithms } & \multicolumn{4}{|c|}{ Cost (\$/day) } & \multirow{2}{*}{ Time (s) } \\
\hline & Best & Worst & Mean & Std(\%) & \\
\hline BSPSO5+DE/rand/1 & 571,421 & 571,948 & 571,615 & 0.031 & 61.8 \\
\hline BSPSO5+DE/current to best/1 & 571,528 & 573,137 & 572,372 & 0.114 & 62.3 \\
\hline BSPSO5+DE/current to best/2 & 571,576 & 571,836 & 571,712 & 0.015 & 72.4 \\
\hline BSPSO5+DE/best/1 & 571,622 & 573,510 & 572,539 & 0.104 & 58.7 \\
\hline BSPSO5+DE/best/2 & 571,738 & 572,669 & 572,122 & 0.048 & 63.1 \\
\hline BSPSO5+DE/rand/2 & 571,436 & 572,098 & 571,606 & 0.045 & 63.4 \\
\hline BSPSO5+SaDE & 571,296 & 571,383 & 571,337 & 0.005 & 60.3 \\
\hline
\end{tabular}

In addition to compare the BSPSO variants performance on the proposed UCSPEV problem, different variants of DE is selected to compare the result. Besides SaDE, 6 DE variants from (Qin and Suganthan, 2005) are 
employed to compare the result performances on the C2 $10 \%$ power reserve scenario, including $D E / r a n d / 1$, $\mathrm{DE} /$ current to best/1, DE/current to best/2, DE/best/1, DE/best/2 and DE/rand/2. The parameter settings $C R$ and $F$ of the algorithms are the same with the beset setting of SaDE as $C R=0.3, F=0.7$ achieved in Table 4. Table 6 shows the best, worst, mean results and the standard deviation of ten independent runs of 7 DE variants hybrid with BSPSO5 on solving the given scenario.

It could be observed from Table 6 that the SaDE based hybrid algorithm outperforms all the other DE variants in seeking the optimum using comparatively low computational cost. The standard deviation of SaDE method is 27.20 , showing significant advantage in obtaining comparatively good solutions. The results comparison of 5 BSPSO based hybrid methods and 7 DE based hybrid methods are shown in figure 6 to 9 , further proves the superior performance of the combination of the hybrid algorithm combination of BSPSO5 and SaDE. The advantage of proposed method BSPSO5 is majorly due to the shape of the particular transfer function match well with the evolutionary process, avoiding the solutions to fell into the local minimums. Comparing to the optimal result $\$ 563,937$ of UC without PEVs scenarios, only around $\$ 8,000$ per day e.g. roughly $1.5 \%$ of the total cost is increased, bringing a proportional cost rise for the additional $411 \mathrm{MWh}$ over the whole day load $27100 \mathrm{MWh}$. This combination is selected as the tool, namely BSPSO-SaDE, to solve the other cases and scenarios for UCSPEV problem in the rest of the paper.

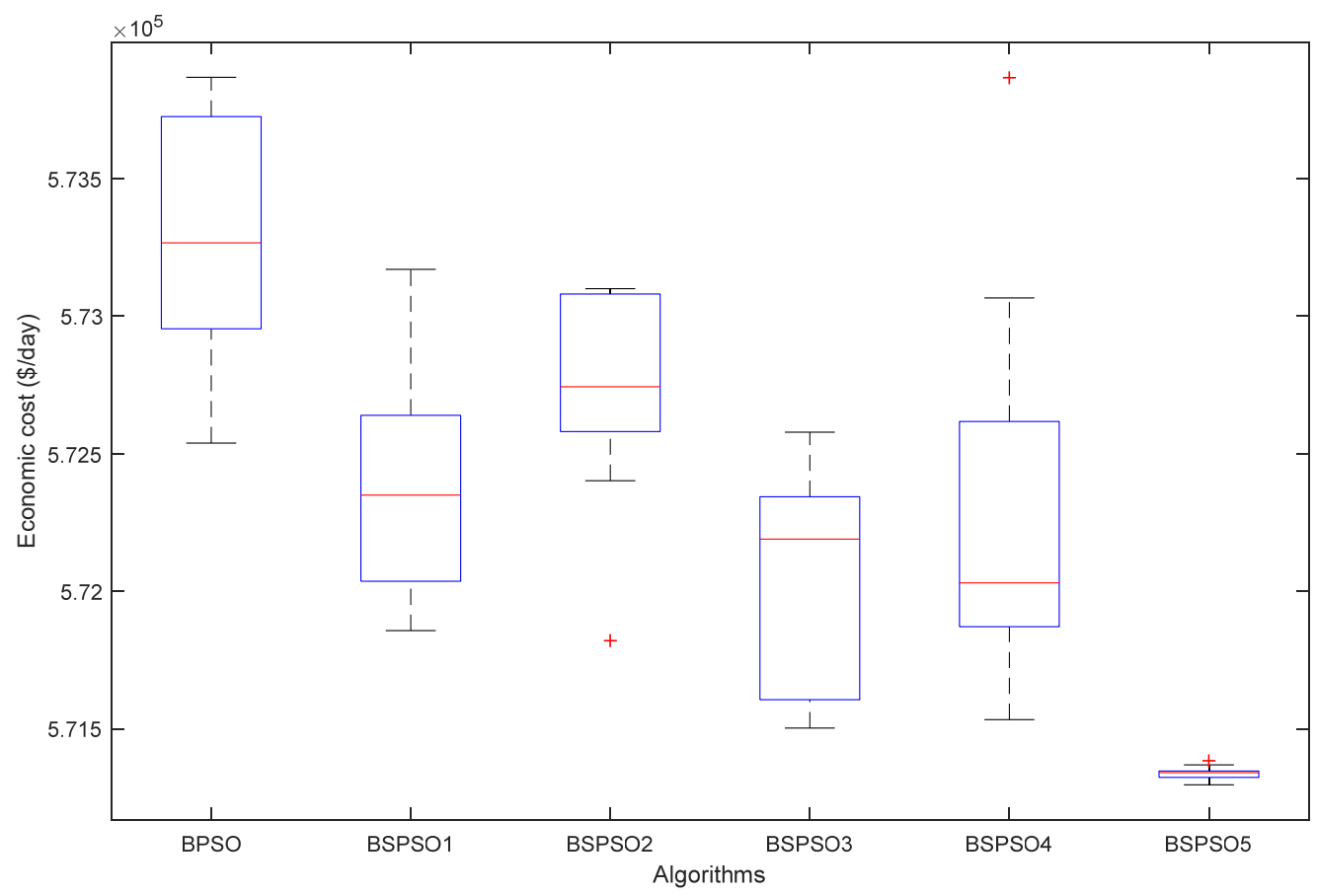

Fig. 6 Results comparison of 5 BSPSO variants on C2 10\% reserve 


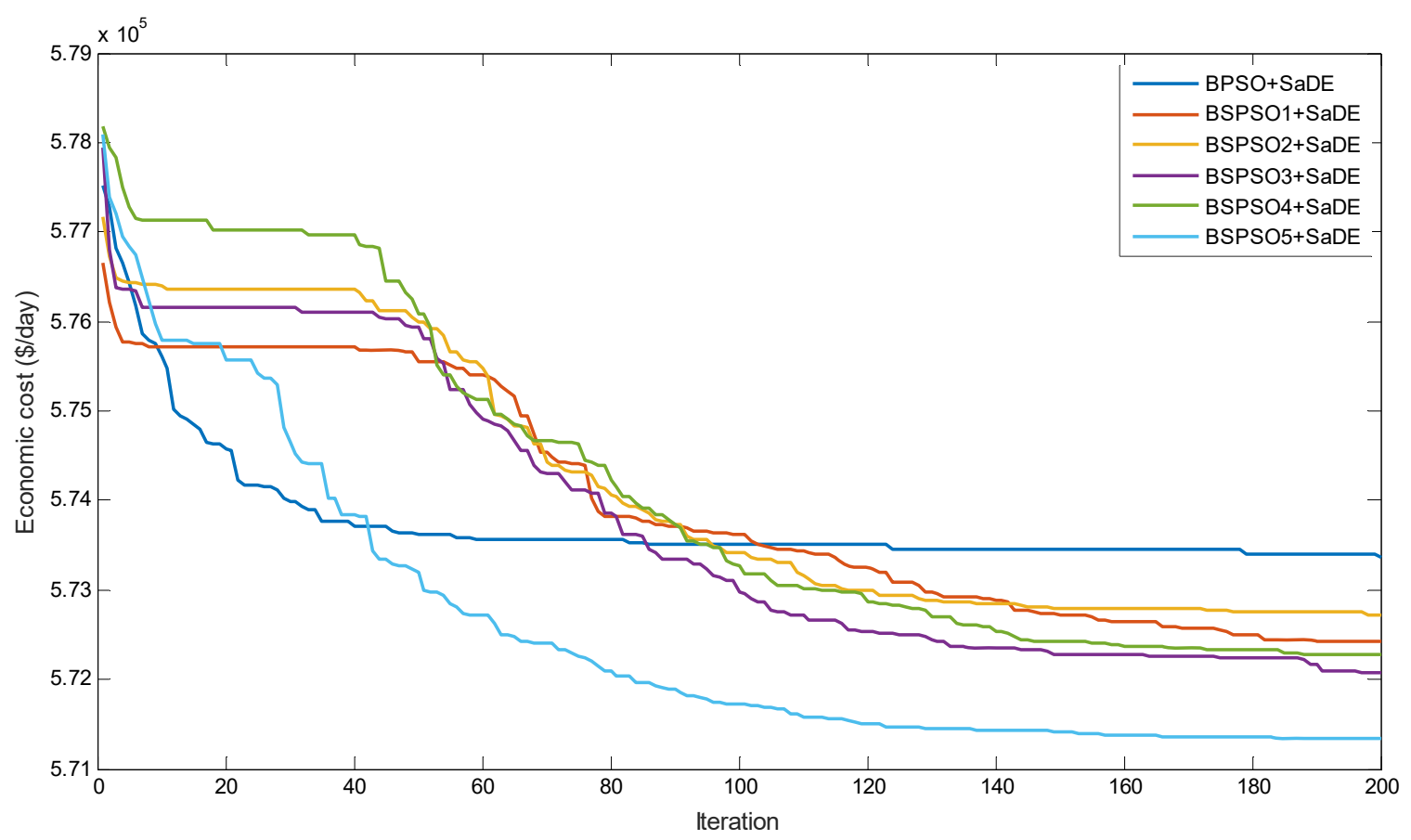

Fig. 7 Converging process of 5 BSPSO variants on C2 10\% reserve

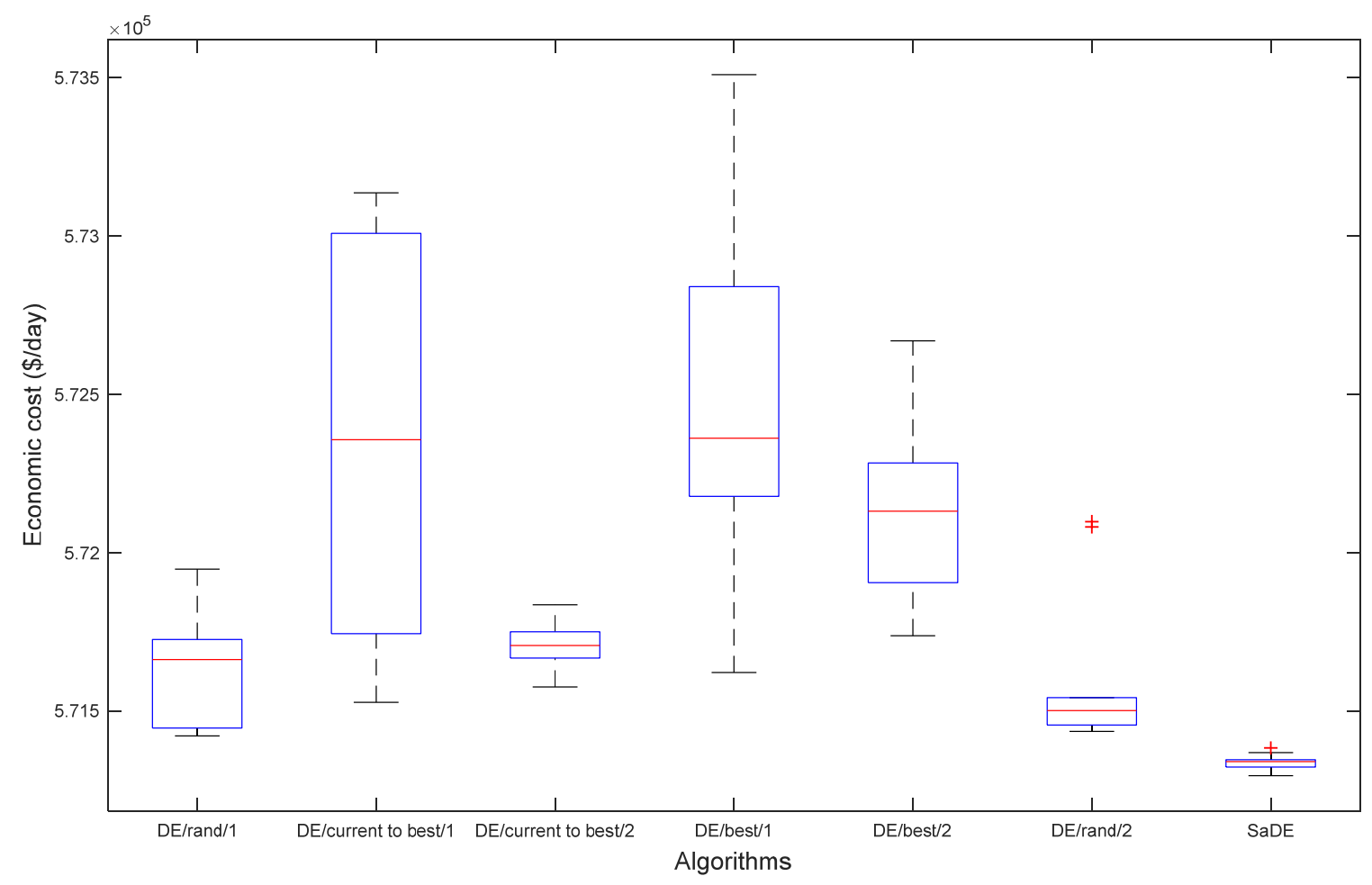

Fig. 8 Results comparison of 7 DE variants on C2 10\% reserve 


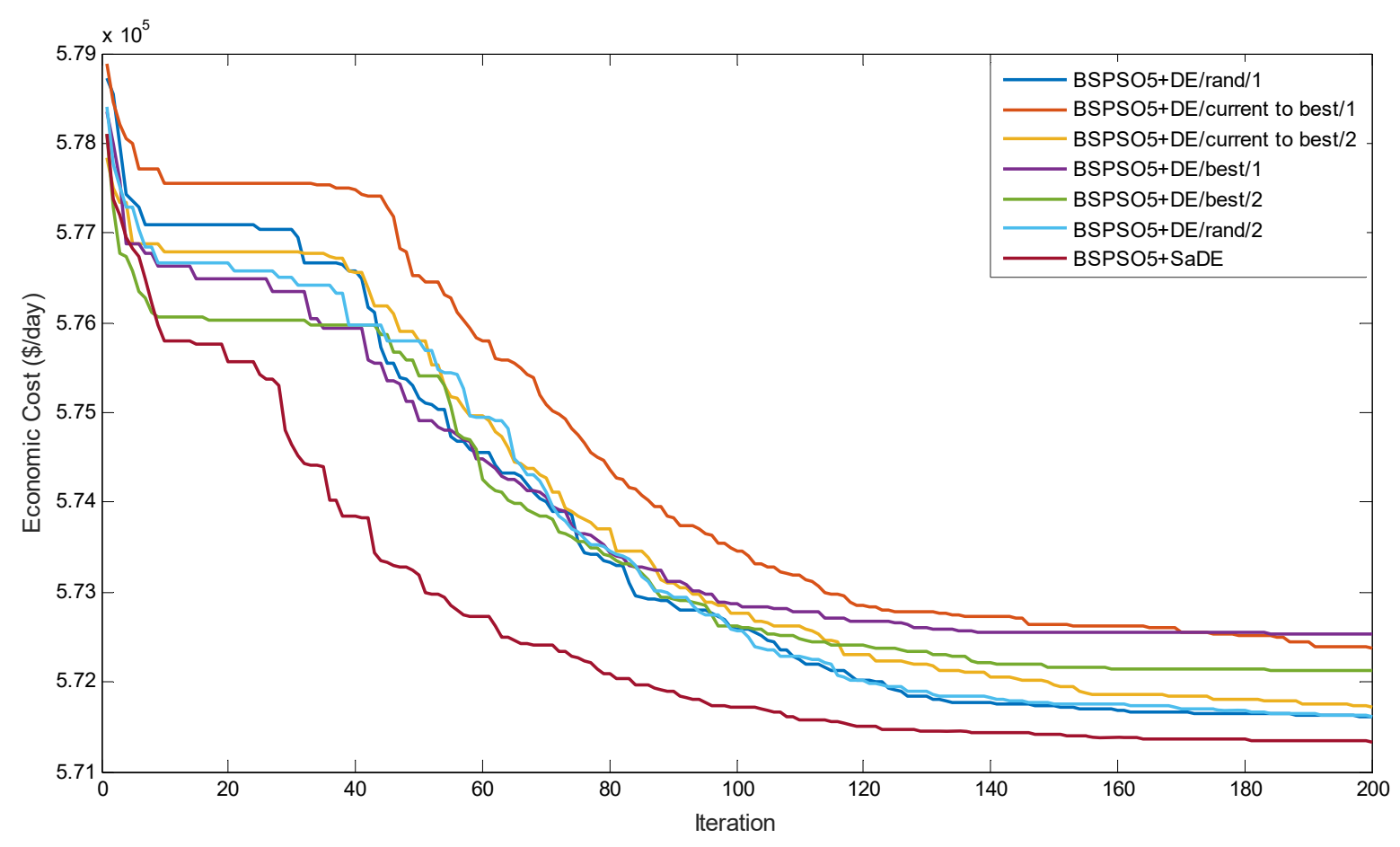

Fig. 9 Converging process of 7 DE variants on C2 10\% reserve

Utilising the aforementioned well-tuned meta-heuristic tool BSPSO5-SaDE, the flexible PEV charging scenarios are discussed in the rest of this section in addition to the $10 \%$ reserve scenario. Three levels of power reserve provided by the thermal generation plants are considered, with $0 \%, 5 \%$ and $10 \%$ respectively, to compare the difference of economic cost and are denoted as Case 2-Scenario 1 (C2-S1) to Case 2-Scenario 3 (C2-S3). Note that in scenarios C2-S1 and C2-S2, the power reserve is only partly or not supported by the thermal generations. The PEVs battery storage and renewable generations are assumed to provide the complementary reserve to ensure the system reliability which illustrated in Figure 1, while the costs of them are ignored in this paper. Two similar load levelling methods using original BPSO and an integer PSO (IPSO), namely average load levelling method (A.Y. Saber and Venayagamoorthy, 2010) and intelligent load levelling method (Saber and Venayagamoorthy, 2011), are tested respectively. The load levelling method evenly assigns the demand of PEVs into a sequence of hours, by which means the fluctuated charging behaviours are well coordinated. The two methods adopt similar system with the same PEVs capacity but different scheduling methods which are appropriate in comparing the efficiency of computational techniques. It should be also noted that both works (A.Y. Saber and Venayagamoorthy, 2010)(Saber and Venayagamoorthy, 2011) did not consider the spinning reserve provided by the 10 unit thermal generation system, both of which fall into the scenario of C2-S1. The 
comparisons of optimization results are illustrated in Table 7 where ' - ' represents that the data is not available from the publications.

Table 7.

Numerical results for UC considering G2V mode

\begin{tabular}{|c|c|c|c|c|c|}
\hline \multirow[b]{2}{*}{ Scenarios of spinning reserve } & \multirow{2}{*}{$\begin{array}{c}\text { BPSO-IPSO } \\
\text { Cost (\$/day) } \\
\text { Average load leveling } \\
\text { (A.Y. Saber and } \\
\text { Venayagamoorthy, } \\
\text { 2010) }\end{array}$} & \multirow{2}{*}{$\begin{array}{c}\text { BPSO-IPSO } \\
\text { Cost (\$/day) } \\
\text { Intelligent load leveling } \\
\text { (Saber and } \\
\text { Venayagamoorthy, 2011) }\end{array}$} & \multicolumn{3}{|c|}{$\begin{array}{l}\text { BSPSO-SaDE } \\
\text { Cost (\$/day) }\end{array}$} \\
\hline & & & Best & Worst & Mean \\
\hline C2-S1: 0\% reserve from thermal generation & 567,845 & 566,898 & 559,652 & 560,318 & 559,953 \\
\hline C2-S2: $5 \%$ reserve from thermal generation & - & - & 565,376 & 565,764 & 565,618 \\
\hline C2-S3: 10\% reserve from thermal generation & - & - & 571,296 & 571,383 & 571,337 \\
\hline
\end{tabular}

Three power reserve scenarios for the UCSPEV problem are optimized by proposed method as shown in Table

7. By comparing with the other two $0 \%$ thermal reserve scheduling results, BSPSO-SaDE achieves the best cost at $559,652 \$ /$ day, which is $8,193 \$ /$ day and $7,246 \$$ /day less than the two BPSO-IPSO based load levelling methods. The difference between the best results and worst results of the three reserve levels scenarios are $666 \$ /$ day, $388 \$ /$ day and $87 \$ /$ day respectively, and the comparison of best results in 10 Trials on all scenarios of Case 2 is shown in figure 10, showing strong searching stability of the proposed algorithm. Similar to the UC only problem test, the BSPSO based hybrid method demonstrates distinguished performance on the UCSPEV problem. This is majorly due to the proper adoption of an efficient transfer function for the binary variables evolution. The strong robustness of the SaDE method successfully associates with the binary based method in determining the PEVs power contribution. Moreover, comparing the best results of the three reserve levels scenarios C2-S1 to C2-S3, C2-S1 has successfully saved $5724 \$$ /day and 11,644 \$/day than C2-S2 and C2-S3. 


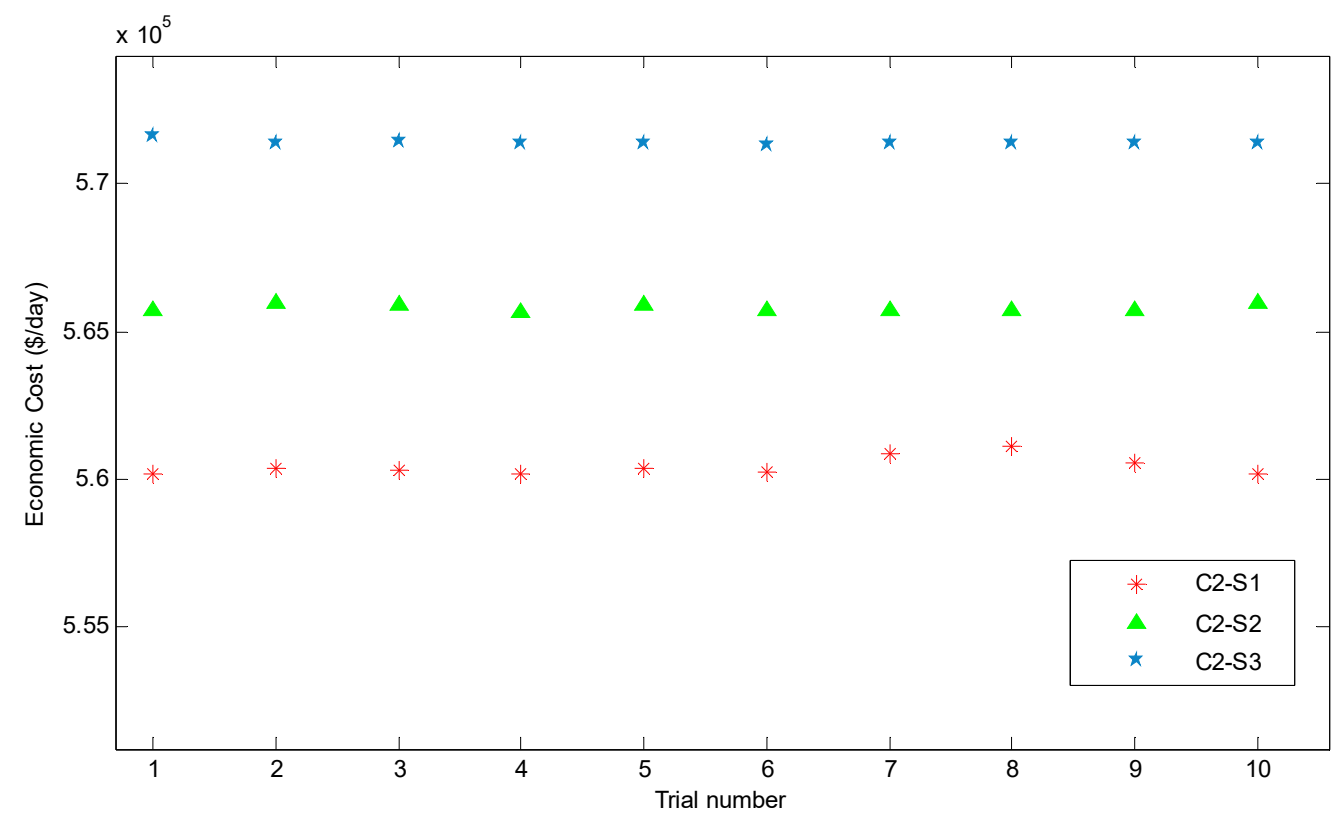

Fig. 10 Comparison of results in 10 Trials of BSPSO-SaDE on Case 2

In the charging-only case, the proposed BSPSO-SaDE algorithm shows remarkable searching ability and outperforms the BPSO method in both solving the UC problem and dispatching the PEV charging load. Three levels of scenarios are compared under different levels of spinning reserve provided by the thermal units. Besides the intelligent charging, V2G service has the potential to further save the economic cost, which will be discussed in the following sub-section 4.3.

\subsection{Case 3: 10 unit with plug-electric vehicles discharging-only}

The other power flow direction is PEV discharge where PEVs inject power back to the grid when necessary. This is referring to V2G service proposed a decade ago (Kempton and Tomić, 2005). The aggregator of PEVs is not only able to act as generator and directly provides power to the grid, but also can offer power reserve. The start-up cost of thermal unit which is used as reserve unit is therefore reduced. Similarly, 50,000 PEVs are integrated into the 10 unit system, and the $50 \%$ state of charge (SOC) of the $15 \mathrm{kWh}$ battery in each PEV is assumed to provide V2G service and get charged back only from renewable energy generations (Ahmed Yousuf Saber and Venayagamoorthy, 2010) (Talebizadeh et al., 2014). The discharging efficiency is $85 \%$ and the total alternative discharging power is calculated as $318.5 \mathrm{MWh}(15 \mathrm{kWh} \times 50000 \times 50 \% \times 0.85)$ (Saber and Venayagamoorthy, 2011). The maximum discharging power is assumed to be $10 \%$ of the total discharging power capacity (Talebizadeh et al., 2014). The priority charging list shown in Table 3 is utilized in a reverse order in the PEV discharging scenario. In another word, the priority list of PEVs discharging is the descending 
sequence of predicted power load. Three levels of power reserve are also adopted in the comparison, namely C3-S1, C3-S2 and C3-S3 with 0\%, 5\% and 10\% reserve provided by thermal units respectively. The system data and optimization parameters are the same as Case 2 and 10 different runs are implemented. The maximum iteration of optimization is also 200. The scheduling results of the BPSO-IPSO method (Ahmed Yousuf Saber and Venayagamoorthy, 2010) and GA-LR method (Talebizadeh et al., 2014) are also listed in Table 8 for performance comparison. The optimisation processes of each scenario are shown in figure 11.

Table 8.

Numerical results for UC considering V2G mode

\begin{tabular}{|c|c|c|c|c|c|c|c|c|c|}
\hline \multirow[t]{2}{*}{ Scenarios of spinning reserve } & \multicolumn{3}{|c|}{$\begin{array}{c}\text { BPSO-IPSO (Ahmed Yousuf } \\
\text { Saber and Venayagamoorthy, } \\
2010) \\
\text { Cost }(\$ / \text { day })\end{array}$} & \multicolumn{3}{|c|}{$\begin{array}{c}\text { GA-LR (Talebizadeh et al., } \\
2014) \\
\text { Cost }(\$ / \text { day) }\end{array}$} & \multicolumn{3}{|c|}{$\begin{array}{l}\text { BSPSO-SaDE } \\
\text { Cost (\$/day) }\end{array}$} \\
\hline & Best & Worst & Mean & Best & Worst & Mean & Best & Worst & Mean \\
\hline $\begin{array}{c}\text { C3-S1: 0\% reserve from thermal } \\
\text { generation }\end{array}$ & - & - & - & - & - & - & 543,311 & 543,936 & 543,546 \\
\hline $\begin{array}{l}\text { C3-S2: } 5 \% \text { reserve from thermal } \\
\text { generation }\end{array}$ & 553,090 & 553,636 & 553,385 & - & - & - & 548,138 & 549,448 & 548,597 \\
\hline $\begin{array}{l}\text { C3-S3: } 10 \% \text { reserve from thermal } \\
\text { generation }\end{array}$ & 559,685 & 560,254 & 560,094 & 552,427 & 553,765 & 552,966 & 551,031 & 551,614 & 551,338 \\
\hline
\end{tabular}

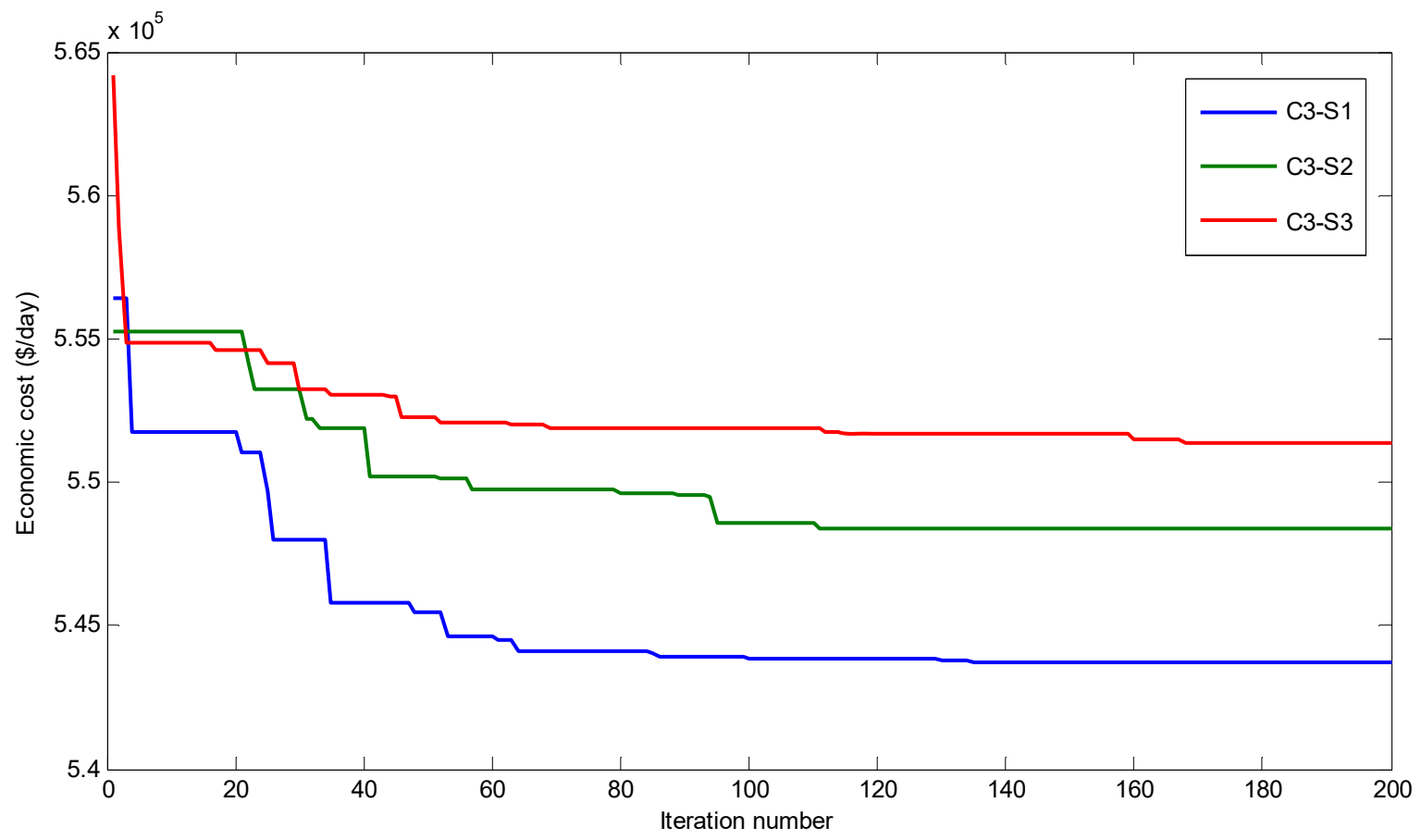

Fig. 11 Optimization processes of three scenarios in C3

The results again show the competitive performance of the proposed method in optimizing the UCSPEV problem. The new method significantly outperforms the two counterparts taking the best, worst and mean 
values into account on all the three scenarios. Both BPSO and GA-LR do not consider the C3-S1 scenarios, where all the power reserve is provided by non-thermal generation. Among the three scenarios of power reserve optimized by BSPSO-SaDE, the $10 \%$ reserve scenario costs $7,720 \$ /$ day and $2,893 \$ /$ day more than scenarios with $0 \%$ thermal reserve and $5 \%$ thermal reserve respectively. Comparing the best results achieved by BPSO-IPSO and BSPSO-SaDE in C3-S2, the new method successfully saves 4,952 \$/day economic cost. Moreover, a significant cost reduction is made by BSPSO-SaDE in C3-S3 that $8,654 \$ /$ day and 1,396 \$day costs are saved, compared with the BPSO and GA-LR methods. The new optimization algorithm combining with the PEV priority list reduced 1396 \$/day economic cost comparing with the results achieved by GA-LR method.

\subsection{Case 4: 10 unit with plug-electric vehicles bidirectional charging}

In the practical PEVs scheduling, the unidirectional scenarios might not be ideal for both system operators and users. Bidirectional charging and discharging provide flexible options for power system operators. In this case, the power system data and optimization parameters are the same as aforementioned scenarios. 50,000 PEVs with $15 \mathrm{kWh}$ battery in each vehicle are again employed and integrated within the 10 unit system, where only 34000 PEVs drivers are willing to provide V2G service, and 50\% SOC, $85 \%$ charging efficiency and $80 \%$ discharging efficiency are assumed. The limit of charging and discharging power is assumed as $20 \%$ of the total battery energy capacity (Talebizadeh et al., 2014). Therefore, the maximum charging power is calculated as $15 \mathrm{kWh} \times 50,000 \times 50 \% \times 85 \% \times 20 \%=63.75 \mathrm{MW}$, and the maximum discharging power is calculated as $15 \mathrm{kWh}$ $\times 34,000 \times 50 \% / 80 \% \times 20 \% \times(-1)=-63.75 \mathrm{MW}$. In addition, the total energy necessity is assumed to be 0 MWh due to that all PEVs act as energy storage for renewable energy and obtain all the energy from nonthermal approaches. Given that the scheduling period unit is 'hour', all the generation capacity, load and results are briefly denoted in MW. However, the total energy necessity along the 24-hour horizon of PEVs has to be denoted as energy format MWh, and this could be denoted as power unit MW once scheduled into the hourly sections. Three scenarios of different power reserve level are studied with 10 independent runs respectively. The statistics results are shown in Table 9, in a comparison with the results in (Talebizadeh et al., 2014). 
Table 9.

Numerical results for UC considering G2V/V2G mode

\begin{tabular}{|c|c|c|c|c|c|c|}
\hline \multirow{2}{*}{ Scenarios of spinning reserve } & \multicolumn{2}{|c|}{$\begin{array}{c}\text { GA-LR (Talebizadeh et al., 2014) } \\
\text { Cost (\$/day) }\end{array}$} & \multicolumn{3}{c|}{$\begin{array}{c}\text { BSPSO-SaDE } \\
\text { Cost (\$/day) }\end{array}$} \\
\cline { 2 - 7 } & Best & Worst & Mean & Best & Worst & Mean \\
\hline C4-S1: 0\% reserve from thermal generation & - & - & - & $\mathbf{5 4 8 , 0 1 7}$ & 548,481 & 548,300 \\
\hline C4-S2: $5 \%$ reserve from thermal generation & - & - & - & $\mathbf{5 5 0 , 2 9 2}$ & 551,114 & 550,767 \\
\hline C4-S3: $10 \%$ reserve from thermal generation & 561,821 & 566,281 & 564,050 & $\mathbf{5 5 6 , 3 4 3}$ & $\mathbf{5 5 7 , 1 2 6}$ & $\mathbf{5 5 6 , 7 4 9}$ \\
\hline
\end{tabular}

It can be observed from Table 9 that the proposed BSPSO-SaDE method achieved a best cost as 556,343 $\$ /$ day, which is 5,478 \$/day lower than the best result achieved by GA-LR in C4-S3. The C4-S1 scenario spends 8,326 $\$ /$ day and 2,275 \$/day more fuel than C4-S3 and C4-S2. The load demand and multiple combinations with three PEV dispatching results are illustrated in figure 12, where C4-S3 shows the strongest ability to reduce peak load during 9:00-15:00 and 20:00-22:00 and move these load to off-peak time 01:00-05:00 and sub-offpeak time 16:00-19:00, showing the strongest ability of PEVs in shifting load demand.

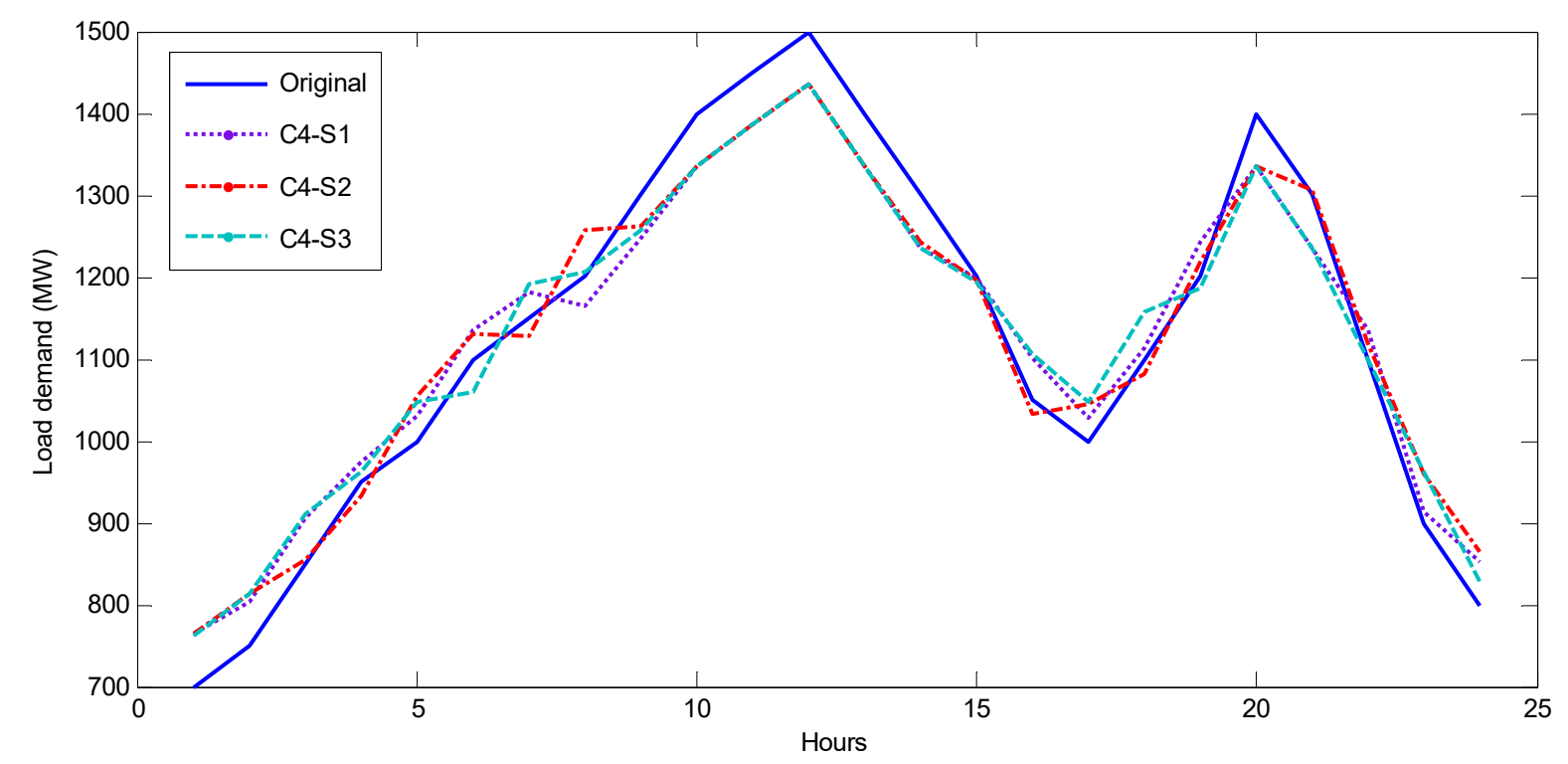

Fig. 12 Load demand and its combinations with three PEVs dispatching results.

\subsection{Cases comparative analysis}

Three flexible PEVs integration modes are implemented in the UCSPEV problem and solved respectively by the proposed meta-heuristic method. The impact of PEV integrations on the conventional UC problem is further analysed in this section by statics comparative study. 
The intelligent PEVs scheduling helps to reduce the number of online units for saving economic cost. The unit committed status changes due to the shifted or additional power demand with PEV integration. Note that it is fair to compare the UC results only under the same thermal power reserve levels. Therefore, unit status changes of all the $10 \%$ power reserve scenarios including C2-S3, C3-S3 and C4-S3 compared with the result of benchmark $\mathrm{C} 1$ are shown in figure 13.

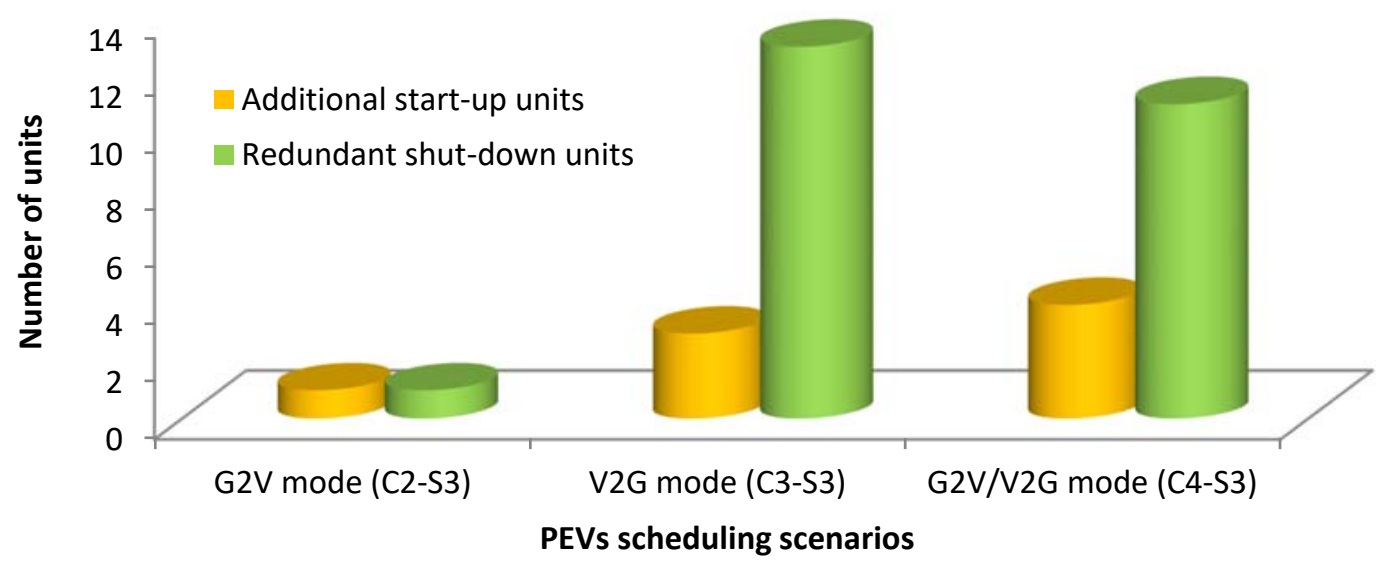

Fig. 13 Unit status change of all 10\% thermal power reserve scenarios

It could be observed first from the figure 13 that only one additional unit is started up associated with a redundant unit is shut down. The extra PEVs charging load is therefore well scheduled without introducing significant commitment deviation. While in the V2G mode, there are 13 units shut down compared with benchmark results with only 3 units are additionally committed. Moreover, it is worth to notice that in the G2V/V2G mode, though the total energy necessity from the grid is zero, 10 unit commitment slots of 5 units (green cells in Table 17) are reduced by the intelligent scheduling of PEVs charging and discharging. The specific dispatch agendas for each unit are accumulated and shown in figure 14. The base load support unit U1 and U2 are online on all 24 hours, whereas the other units are various. The unit U3-U6 have been online in all the cases where the comparatively cheaper unit U5 is shown to be in a prior sequence. Moreover, U7 is committed 10 hours in the first two cases but completely off-line in C3-S3 case. The online hours of expensive units U8 U9 and U10 are significant reduced by comparing the $10 \%$ thermal reserve scenarios C1, C2-S3, C3-S3 and C4-S3. The G2V/V2G mode C4-S3 successfully avoids committing U10 and reduces the committing hours of U7-U9. Similar results could be observed in the comparisons of S1 and S2 along the cases C2 to C4. 


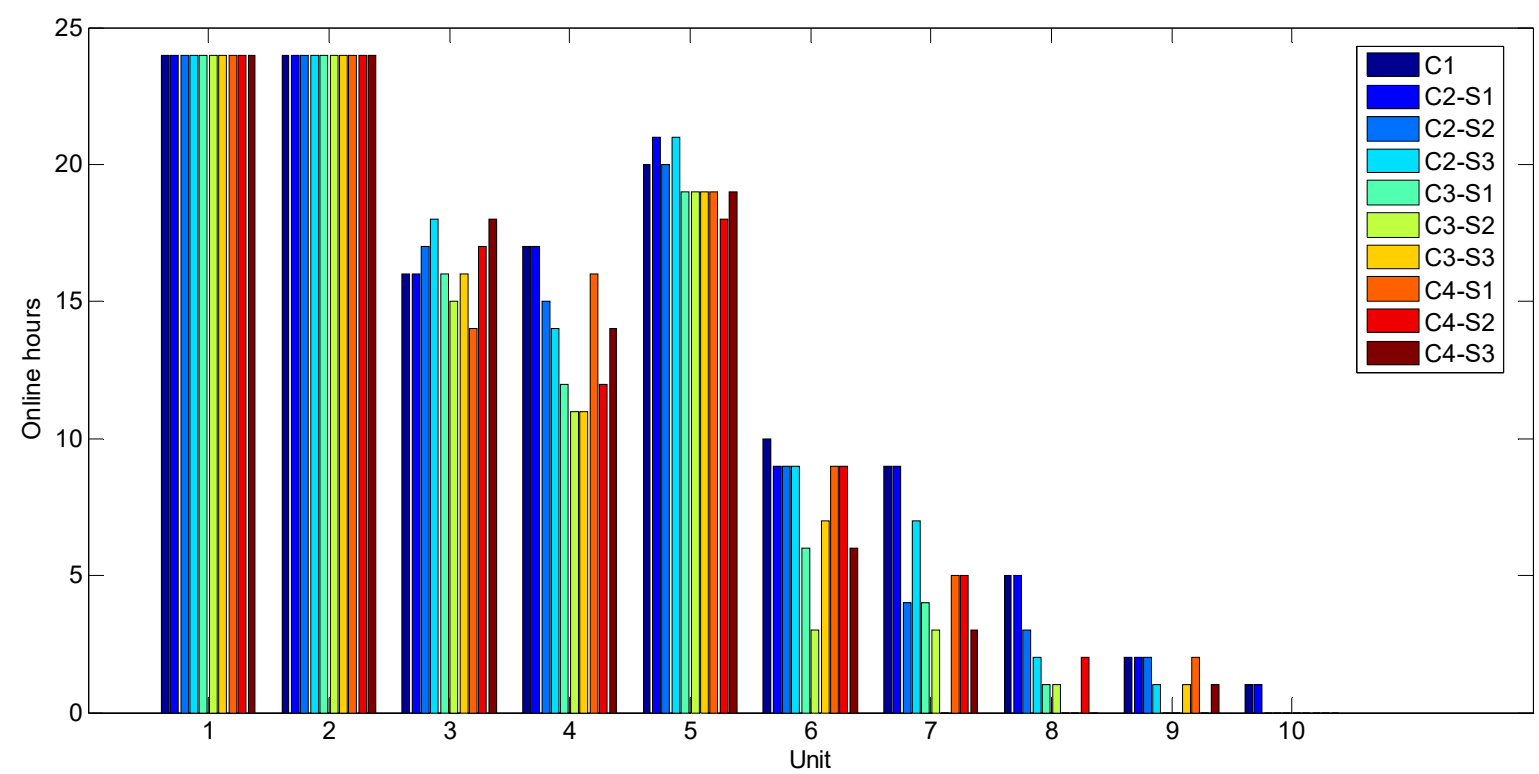

Fig. 14 Number of online hours of each thermal unit for various units

\section{Conclusion}

In this paper, a new complex UCSPEV problem is formulated to simultaneously determine the day-ahead unit commitment and power scheduling for PEVs aggregators. A novel hybrid meta-heuristic method is proposed to solve the problem combines the advantages of binary symmetric PSO, SaDE and lambda iteration method. The superior performance of the new symmetric hybrid method was validated using the standard 10-unit dayahead commitment task and shown to be an efficient method in dealing with all power reserve and flexible charging and discharging cases of UCSPEV relevant problems, outperforming its predecessors by achieving more appropriate UC and PEV power input/output. It therefore provides a powerful tool to intelligently dispatch PEV charging/discharging power in cooperation with UC for cost minimization. In addition, the strategies of utilizing V2G service and intelligently dispatching of G2V/V2G were proved to remarkably save the economic cost as flexible energy storage.

With the fast development of renewable energy generation, PEVs and other new type of energy storages in power system, the implementation of smart grid calls for more computational tools to reduce the economic cost, environmental cost as well as to maximize the user welfare. Latest meta-heuristic methods provide more options for power engineers to intelligently operate and gain smartness for the power system. On the other hand, the mass roll out of PEVs is of significant potentials in participating ancillary services such as reserve and frequency regulations, which may lead to the future work of this study. 


\section{Acknowledgement}

This research is financially supported by China NSFC under grants 51607177, 61773252, 61433012, U1435215,

China Postdoctoral Science Foundation (2018M631005), Natural Science Foundation of Guangdong Province under grants 2018A030310671 and UK EPSRC grant under the Optimising Energy Management in Industry 'OPTEMIN' project EP/P004636/1.

\section{Reference}

Afkousi-Paqaleh, M., Rashidinejad, M., Pourakbari-Kasmaei, M., 2010. An implementation of harmony search algorithm to unit commitment problem. Electr. Eng. 92, 215-225. https://doi.org/10.1007/s00202-010-0177-z

Akhlaghi, M., Emami, F., Nozhat, N., 2014. Binary TLBO algorithm assisted for designing plasmonic nano bi-pyramids-based absorption coefficient. J. Mod. Opt. 61, 1092-1096. https://doi.org/10.1080/09500340.2014.920537

Chakraborty, S., Ito, T., Senjyu, T., Saber, A.Y., 2012. Unit commitment strategy of thermal generators by using advanced fuzzy controlled binary particle swarm optimization algorithm. Int. J. Electr. Power Energy Syst. 43, 1072-1080. https://doi.org/10.1016/j.ijepes.2012.06.014

Chakraborty, S., Senjyu, T., Yona, A., Funabashi, T., 2011. Fuzzy quantum computation based thermal unit commitment strategy with solar-battery system injection, in: 2011 IEEE International Conference on Fuzzy Systems (FUZZ-IEEE 2011). Presented at the 2011 IEEE International Conference on Fuzzy Systems (FUZZ-IEEE 2011), pp. 2606-2613. https://doi.org/10.1109/FUZZY.2011.6007521

Chan, C.C., 2007. The State of the Art of Electric, Hybrid, and Fuel Cell Vehicles 704-718.

Chen, D., Zou, F., Li, Z., Wang, J., Li, S., 2015. An improved teaching-learning-based optimization algorithm for solving global optimization problem. Inf. Sci. 297, 171-190. https://doi.org/10.1016/j.ins.2014.11.001

Chen, P.-H., 2012. Two-Level Hierarchical Approach to Unit Commitment Using Expert System and Elite PSO. IEEE Trans. Power Syst. 27, 780-789. https://doi.org/10.1109/TPWRS.2011.2171197

Chung, C.Y., Yu, H., Wong, K.-P., 2011. An Advanced Quantum-Inspired Evolutionary Algorithm for Unit Commitment. IEEE Trans. Power Syst. 26, 847-854. https://doi.org/10.1109/TPWRS.2010.2059716

Clement-Nyns, K., Haesen, E., Driesen, J., 2010. The Impact of Charging Plug-In Hybrid Electric Vehicles on a Residential Distribution Grid 371-380.

Dallinger, D., Wietschel, M., 2012. Grid integration of intermittent renewable energy sources using price-responsive plug-in electric vehicles 3370-3382.

Das, S., Suganthan, P.N., 2011. Differential Evolution: A Survey of the State-of-the-Art. IEEE Trans. Evol. Comput. 15, 4-31. https://doi.org/10.1109/TEVC.2010.2059031

Datta, D., 2013. Unit commitment problem with ramp rate constraint using a binary-real-coded genetic algorithm. Appl. Soft Comput. 13, 3873-3883. https://doi.org/10.1016/j.asoc.2013.05.002

Deilami, S., Masoum, A.S., Moses, P.S., Masoum, M.A.S., 2011. Real-Time Coordination of Plug-In Electric Vehicle Charging in Smart Grids to Minimize Power Losses and Improve Voltage Profile 456-467.

Foley, A., Tyther, B., Calnan, P., Ó Gallachóir, B., 2013. Impacts of Electric Vehicle charging under electricity market operations 93-102. 
Gaing, Z.-L., 2003. Discrete particle swarm optimization algorithm for unit commitment, in: IEEE Power Engineering Society General Meeting, 2003. Presented at the IEEE Power Engineering Society General Meeting, 2003, p. 424 Vol. 1. https://doi.org/10.1109/PES.2003.1267212

Jeong, Y.-W., Park, J.-B., Jang, S.-H., Lee, K.Y., 2010. A New Quantum-Inspired Binary PSO: Application to Unit Commitment Problems for Power Systems. IEEE Trans. Power Syst. 25, 1486-1495. https://doi.org/10.1109/TPWRS.2010.2042472

Ji, B., Yuan, X., Chen, Z., Tian, H., 2014. Improved gravitational search algorithm for unit commitment considering uncertainty of wind power. Energy 67, 52-62. https://doi.org/10.1016/j.energy.2014.02.014

Jiang, Q., Zhou, B., Zhang, M., 2013. Parallel augment Lagrangian relaxation method for transient stability constrained unit commitment. IEEE Trans. Power Syst. 28, 1140-1148. https://doi.org/10.1109/TPWRS.2012.2216553

Jin, C., Tang, J., Ghosh, P., 2013. Optimizing Electric Vehicle Charging With Energy Storage in the Electricity Market. leee Trans. Smart Grid 4, 311-320. https://doi.org/10.1109/TSG.2012.2218834

Kamboj, V.K., Bath, S.K., Dhillon, J.S., 2015. Hybrid HS-random search algorithm considering ensemble and pitch violation for unit commitment problem. Neural Comput. Appl. 1-26. https://doi.org/10.1007/s00521-015-2114-6

Kazarlis, S.A., Bakirtzis, A.G., Petridis, V., 1996. A genetic algorithm solution to the unit commitment problem. IEEE Trans. Power Syst. 11, 83-92. https://doi.org/10.1109/59.485989

Kempton, W., Tomić, J., 2005. Vehicle-to-grid power fundamentals: Calculating capacity and net revenue $268-279$.

Khanmohammadi, S., Amiri, M., Haque, M.T., 2010. A new three-stage method for solving unit commitment problem. Energy 35, 3072-3080. https://doi.org/10.1016/j.energy.2010.03.049

Khodayar, M.E., Wu, L., Shahidehpour, M., 2012. Hourly Coordination of Electric Vehicle Operation and Volatile Wind Power Generation in SCUC. leee Trans. Smart Grid 3, 1271-1279. https://doi.org/10.1109/TSG.2012.2186642

Liu, C., Wang, J., Botterud, A., Zhou, Y., Vyas, A., 2012. Assessment of Impacts of PHEV Charging Patterns on Wind-Thermal Scheduling by Stochastic Unit Commitment. leee Trans. Smart Grid 3, 675-683. https://doi.org/10.1109/TSG.2012.2187687

Mirjalili, S., Lewis, A., 2013. S-shaped versus V-shaped transfer functions for binary Particle Swarm Optimization. Swarm Evol. Comput. 9, 1-14. https://doi.org/10.1016/j.swevo.2012.09.002

Pavić, I., Capuder, T., Kuzle, I., 2015. Value of flexible electric vehicles in providing spinning reserve services. Appl. Energy 157, 60-74. https://doi.org/10.1016/j.apenergy.2015.07.070

Qin, A.K., Suganthan, P.N., 2005. Self-adaptive differential evolution algorithm for numerical optimization, in: The 2005 IEEE Congress on Evolutionary Computation, 2005. Presented at the The 2005 IEEE Congress on Evolutionary Computation, 2005, p. 1785-1791 Vol. 2. https://doi.org/10.1109/CEC.2005.1554904

Quan, H., Srinivasan, D., Khambadkone, A.M., Khosravi, A., 2015. A computational framework for uncertainty integration in stochastic unit commitment with intermittent renewable energy sources. Appl. Energy 152, 71-82. https://doi.org/10.1016/j.apenergy.2015.04.103

Quan, H., Srinivasan, D., Khosravi, A., 2016. Integration of renewable generation uncertainties into stochastic unit commitment considering reserve and risk: A comparative study. Energy 103, 735-745. https://doi.org/10.1016/j.energy.2016.03.007

Roy, P.K., 2013. Solution of unit commitment problem using gravitational search algorithm. Int. J. Electr. Power Energy Syst. 53, 85-94. https://doi.org/10.1016/j.ijepes.2013.04.001

Saber, A.Y., Venayagamoorthy, G.K., 2012. Resource Scheduling Under Uncertainty in a Smart Grid With Renewables and Plug-in Vehicles 103-109.

Saber, A.Y., Venayagamoorthy, G.K., 2011. Plug-in Vehicles and Renewable Energy Sources for Cost and Emission Reductions 1229-1238. 
Saber, Ahmed Yousuf, Venayagamoorthy, G.K., 2010. Intelligent unit commitment with vehicle-togrid -A cost-emission optimization 898-911.

Saber, A.Y., Venayagamoorthy, G.K., 2010. Efficient Utilization of Renewable Energy Sources by Gridable Vehicles in Cyber-Physical Energy Systems. IEEE Syst. J. 4, 285-294. https://doi.org/10.1109/JSYST.2010.2059212

Sadati, N., Hajian, M., Zamani, M., 2007. Unit Commitment Using Particle Swarm-Based-Simulated Annealing Optimization Approach, in: 2007 IEEE Swarm Intelligence Symposium. Presented at the 2007 IEEE Swarm Intelligence Symposium, pp. 297-302. https://doi.org/10.1109/SIS.2007.367951

Sanchez-Martin, P., Lumbreras, S., Alberdi-Alen, A., 2015. Stochastic Programming Applied to EV Charging Points for Energy and Reserve Service Markets. IEEE Trans. Power Syst. PP, 1-8. https://doi.org/10.1109/TPWRS.2015.2405755

Shukla, A., Singh, S.N., 2016. Advanced three-stage pseudo-inspired weight-improved crazy particle swarm optimization for unit commitment problem. Energy 96, 23-36. https://doi.org/10.1016/j.energy.2015.12.046

Snyder, W.L., Powell, H.D., Rayburn, J.C., 1987. Dynamic Programming Approach to Unit Commitment. IEEE Trans. Power Syst. 2, 339-348. https://doi.org/10.1109/TPWRS.1987.4335130

Sundstrom, O., Binding, C., 2012. Flexible Charging Optimization for Electric Vehicles Considering Distribution Grid Constraints. IEEE Trans. Smart Grid 3, 26-37. https://doi.org/10.1109/TSG.2011.2168431

Talebizadeh, E., Rashidinejad, M., Abdollahi, A., 2014. Evaluation of plug-in electric vehicles impact on cost-based unit commitment. J. Power Sources 248, 545-552. https://doi.org/10.1016/j.jpowsour.2013.09.009

Ting, T.O., Rao, M.V.C., Loo, C.K., 2006. A novel approach for unit commitment problem via an effective hybrid particle swarm optimization. IEEE Trans. Power Syst. 21, 411-418. https://doi.org/10.1109/TPWRS.2005.860907

Trivedi, A., Srinivasan, D., Biswas, S., Reindl, T., 2015. Hybridizing genetic algorithm with differential evolution for solving the unit commitment scheduling problem. Swarm Evol. Comput. 23, 50-64. https://doi.org/10.1016/j.swevo.2015.04.001

Wang, J., Liu, C., Ton, D., Zhou, Y., Kim, J., Vyas, A., 2011. Impact of plug-in hybrid electric vehicles on power systems with demand response and wind power 4016-4021.

White, C.D., Zhang, K.M., 2011. Using vehicle-to-grid technology for frequency regulation and peakload reduction 3972-3980.

Yang, Z., Li, K., Foley, A., 2015. Computational scheduling methods for integrating plug-in electric vehicles with power systems: A review. Renew. Sustain. Energy Rev. 51, 396-416. https://doi.org/10.1016/j.rser.2015.06.007

Yang, Z., Li, K., Niu, Q., Xue, Y., Foley, A., 2014. A self-learning TLBO based dynamic economic/environmental dispatch considering multiple plug-in electric vehicle loads. J. Mod. Power Syst. Clean Energy 2, 298-307. https://doi.org/10.1007/s40565-014-0087-6

Yuan, X., Ji, B., Zhang, S., Tian, H., Hou, Y., 2014. A new approach for unit commitment problem via binary gravitational search algorithm. Appl. Soft Comput. 22, 249-260. https://doi.org/10.1016/j.asoc.2014.05.029

Yuan, X., Nie, H., Su, A., Wang, L., Yuan, Y., 2009. An improved binary particle swarm optimization for unit commitment problem. Expert Syst. Appl. 36, 8049-8055. https://doi.org/10.1016/j.eswa.2008.10.047

Yuan, X., Su, A., Nie, H., Yuan, Y., Wang, L., 2011. Unit commitment problem using enhanced particle swarm optimization algorithm. Soft Comput. 15, 139-148. https://doi.org/10.1007/s00500010-0541-y 
Zhang, J., Tang, Q., Chen, Y., Lin, S., 2016. A hybrid particle swarm optimization with small population size to solve the optimal short-term hydro-thermal unit commitment problem. Energy 109, 765-780. https://doi.org/10.1016/j.energy.2016.05.057 